\title{
Classification Criteria and Clinical Practice Guidelines for Rheumatic Diseases
}

\author{
Rola Hassan, Hanan Faruqui, Reem Alquraa, \\ Ayman Eissa, Fatma Alshaiki, \\ and Mohamed Cheikh
}

\subsection{Introduction}

Rheumatic diseases have many classification criteria and management guidelines that are continuously being updated in order to improve the quality of healthcare provision. With these everevolving criteria and guidelines, practicing clinicians need an easy way to get to the core of these updates and to retain them in an easy and memorable way. Classification criteria are meant to differentiate between similar diseases and also to confirm or rule out a certain disease based on inclusion and exclusion criteria. The diagnosis of rheumatic diseases can be challenging since many clinical signs and symptoms as well as many laboratory markers are not specific and can be positive in many diseases.

There is an important concept that should be addressed. It is that the use of these criteria is meant to be a guide rather than a sole diagnostic tool. It is an established practice that the diagno-

\footnotetext{
R. Hassan $(\bowtie)$

Saudi Commission for Health Specialties, Riyadh, Saudi Arabia

H. Faruqui · R. Alquraa · A. Eissa

M. Cheikh

Doctor Soliman Fakeeh Hospital,

Jeddah, Saudi Arabia

e-mail: mohamed.m.cheikh@gmail.com

F. Alshaiki

Department of Medicine, King Abdulaziz Hospital and Oncology Center, Jeddah, Saudi Arabia
}

sis of rheumatic diseases relies heavily on clinical grounds. The importance of basic skills in rheumatology, namely, the comprehensive history and meticulous musculoskeletal (MSK) examination, cannot be overemphasized. This is obvious as there are no specific diagnostic tests for most rheumatic diseases. For this reason, it is essential to correlate the findings in history and MSK examination with laboratory, radiological, and sometimes histopathological findings to establish the diagnosis.

Guidelines and recommendations for the management of rheumatic diseases were developed to provide guidance based on the best available evidence. It cannot be applied in all situations since the availability of the equipment and medications as well as the patient's condition and ability plays a major role in the application of these guidelines. The physician has to apply the recommendations and guidelines in light of available circumstances and local health authorities' instructions. Such multifaceted decision-making may result in different guideline groups giving different strengths of recommendations for the same treatment. Therefore, all types of evidence, including evidence-based guidelines, need to be examined with care and common sense.

In this section, we will explain how to use the classification criteria for rheumatic diseases in clinical practice, the importance of having classification criteria and the advantage of updating the old ones. We will also cover the most recent 
guidelines and recommendations for the management of the most common rheumatic diseases. This will be aided by the use of tables, graphs, and figures for simplification purposes to help in their application in research and clinical practice and to enhance the accessibility and practicality of this section.

\subsection{Rheumatoid Arthritis Classification Criteria and Management Guidelines}

\subsubsection{Classification Criteria} (Fig. 25.1)

Rheumatoid arthritis (RA) is the most common inflammatory arthritis. If left without treatment, RA can result in joint damage and functional disability. It is diagnosed clinically after the exclusion of other diseases if the symptoms and signs are suggestive. It should be suspected if patients present with inflammatory polyarthritis, after which a detailed history and physical examination, along with appropriate laboratory tests, will help in aiding or excluding this diagnosis.

The initial American College of Rheumatology (ACR) classification criteria for RA were developed in 1987. It was based on patients with established disease. To classify as having RA, the presence of four out of the seven items and the presence of symptoms for more than 6 weeks are required. There was limited practical value in this classification especially for diagnosing early disease. This limitation was corrected in the new (2010) ACR/EULAR (European League Against Rheumatism) classification criteria [1] which was formulated to increase the specificity and sensitivity in diagnosing early RA. These criteria take prognostic markers into account, which were not included in its predecessor. Patients can be identified earlier and started promptly on treat-

\section{Diagnosis of Rheumatoid Arthritis}

a score of $6 / 10$ is needed to definite diagnosis of RA
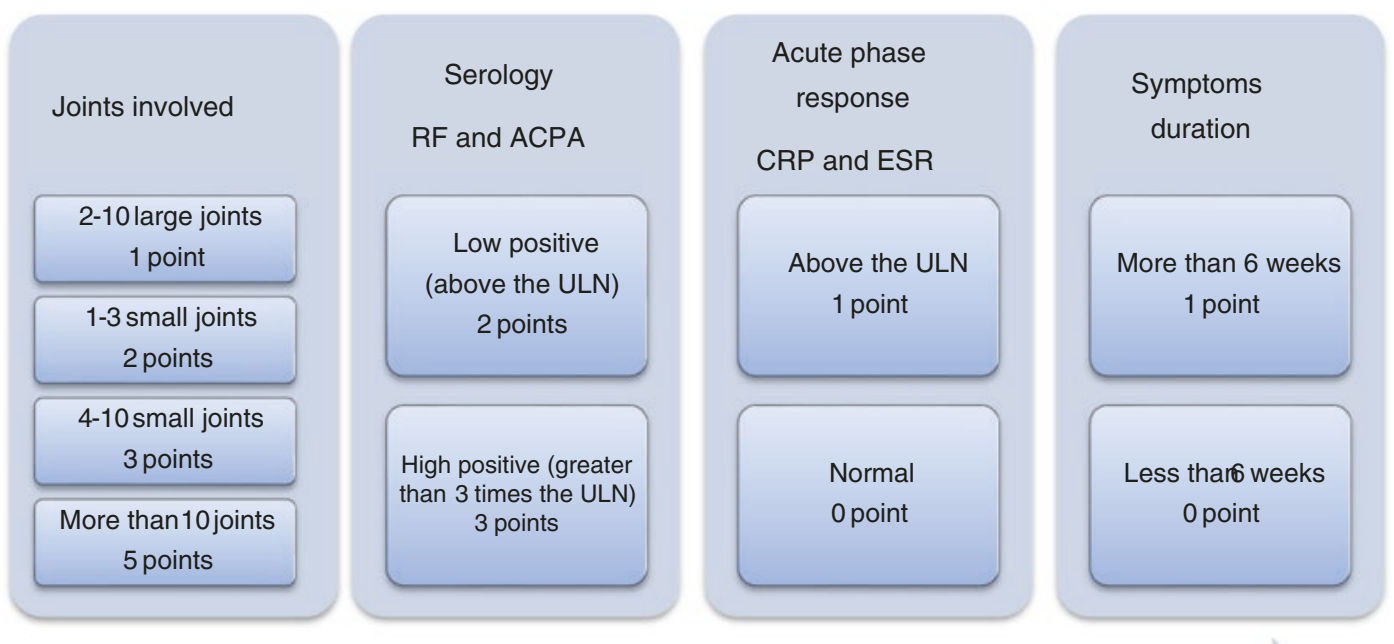

- Erosive disease typical of RA with a history of prior fulfillment of the criteria above

OR

- Longstanding disease with a history of prior fulfillment of the criteria above

Fig. 25.1 Rheumatoid arthritis new classification criteria 
ment with disease-modifying antirheumatic drugs (DMARDs), or they can be entered into clinical trials for promising new agents. It is the hope that these new criteria will help rheumatologists to care for patients with early arthritis and to tailor treatment according to their needs.

\subsubsection{Management Guidelines [2]} (Fig. 25.2)

The general approach to RA treatment has evolved remarkably in the last 10 years as there are an increasing number of effective DMARDs. The early introduction of DMARDs has become standard of care and depends upon early diagnosis that is facilitated by the 2010 ACR/EULAR classification criteria of RA to reach the target remission or in the very least low disease activity.

The 2013 International Task Force recommendations were designed to be based on evidence. They determined 14 recommendations instead of the 15 that were mentioned in the previous 2010 paper, published by the same institute. These recommendations addressed the use of methotrexate or a combination of DMARDs in the first phase, the use of anti-TNF or abatacept or tocilizumab in the second phase, and the use of tofacitinib after the use of at least one DMARD in the third phase.

$$
\text { Both } 2010 \text { and } 2013 \text { guidelines agreed on: }
$$

- The use of low-dose steroids initially.

- Early treatment with DMARD within the first 3-6 months and assessment every 3-6 months.

- Adjustment of treatment according to disease activity scales using the treat-to-target approach.

We have to consider the following in the application of the guidelines:

- Presence of poor prognostic factors.

- Presence of contraindications to methotrexate or other agents.

- The aim is to control the disease and to reach remission or low disease activity.

The 2016 update in the EULAR recommendations for the management of rheumatoid arthritis with synthetic and biological disease-modifying antirheumatic drugs Fig. 25.2.

\section{Management of Rheumatoid Arthritis}

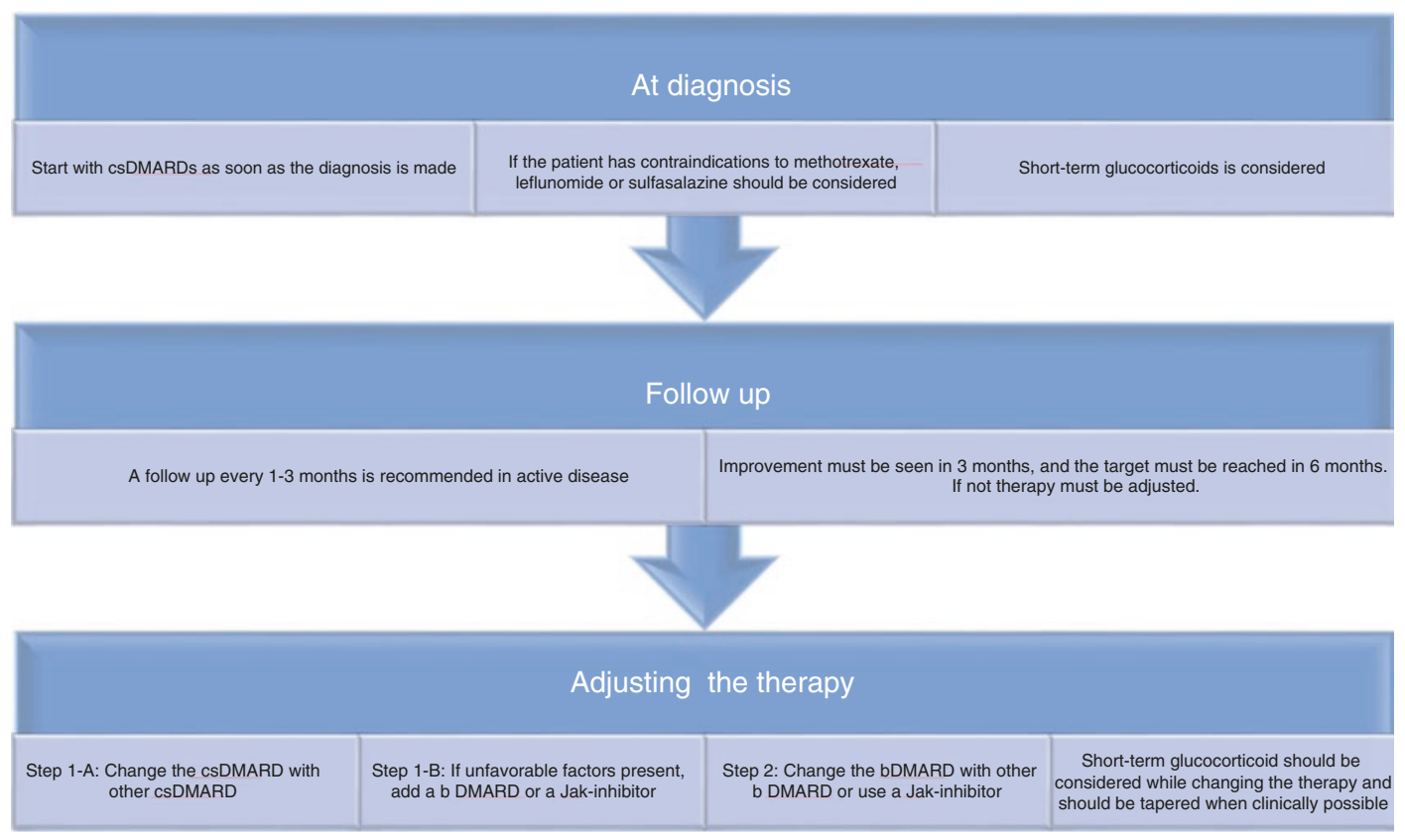

Fig. 25.2 Management of rheumatoid arthritis 


\subsection{Systemic Lupus}

Erythematosus Classification

Criteria and Management

Guidelines

\subsubsection{Classification Criteria of Systemic Lupus \\ Erythematosus (Fig. 25.3)}

Systemic lupus erythematosus (SLE) is a multisystem disease that affects nearly every organ in the body. It can present with a wide array of clini- cal symptoms and signs and with variable disease courses. If left untreated in its early stages, the disease carries significant morbidity and mortality rates.

The Systemic Lupus International Collaborating Clinics (SLICC) represents a consensus group of SLE experts, who amended and validated the 1997 American College of Rheumatology classification criteria in 2012, to address many of the former's limitations (e.g., patients with biopsy-proven lupus nephritis still fail to fulfill the 1997 criteria). The first version

\section{SLE}

Fulfil 4/17 criteria with at least 1 clinical and 1 immunologic OR

- Biopsy proven lupus nephritis in the presence of +ve ANA or anti dsDNA
- Malar rash

- Bullous rash

- Maculopapular rash

- Photosensitive rash

o Toxic epidermal necrolysis (variant of SLE)
Subacute cutaneous lupus erythematosus

(they are erythematous, annular/polycyclic papulosquamous lesions that are photosensitive and resemble psoriasis, they resolve leaving hypo/hyperpigmentation)

\section{- Classic discoid rash \\ o Hypertrophic verrucous lupus \\ - Lupus panniculitis \\ - Mucosal lupus \\ o Lupus erythematosus tumidus \\ o Chilblains lupus}
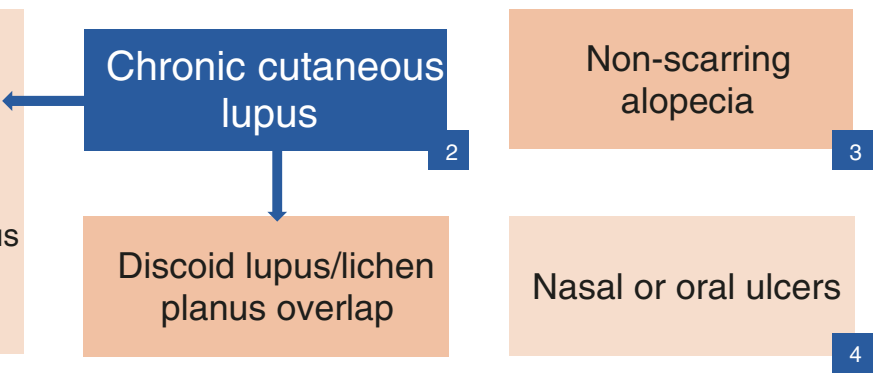

Serous involvement Pleuritis \&/OR Pericarditis

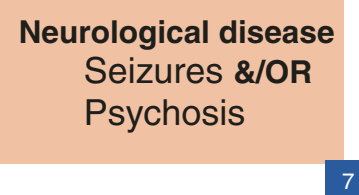

Fig. 25.3 The 2012 SLE SLICC criteria [4]. 


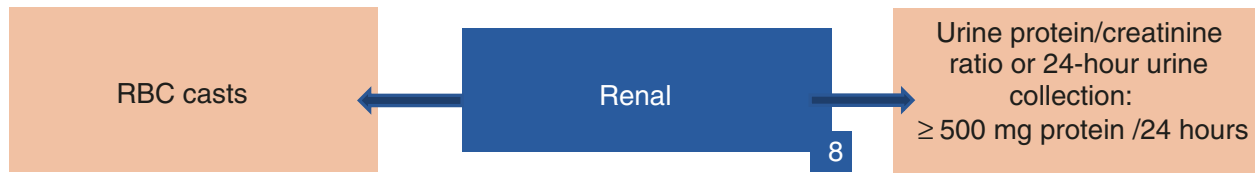

Hematology

WBC $<4000 / \mathrm{mm}^{3}$ Lymphocytes $<1500 / \mathrm{mm}^{3}$

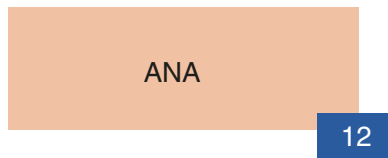

Direct Coombs test (in the absence of hemolytic anemia)

\section{Immunology}

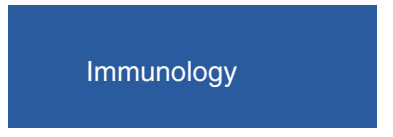

DSDNA

13

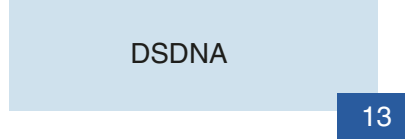

Anti-phospholipid Ab

16
Anti Sm

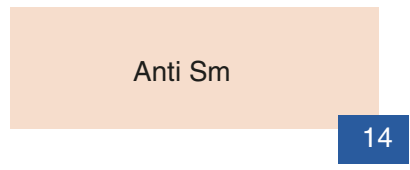

Low complements

\section{SOURCE: THE 2012 SLICC CRITERIA FOR DIAGNOSIS OF SLE}

Fig. 25.3 (continued)

of the ACR criteria was introduced in 1982; it was last updated in 1997. These new criteria (SLICC) had greater sensitivity when compared to the 1997 criteria (97\% vs $83 \%$ ) but lower specificity ( $84 \%$ vs $96 \%$ ) [3, 4]. Both the ACR and the SLICC criteria were initially developed as a way to categorize patients for research purposes, as its use by clinicians for diagnosis is limited by its imperfect sensitivity and specificity.
The 2012 SLICC criteria require the fulfillment of 4 out of the 17 criteria with a minimum of at least one clinical and one immunologic criterion. Lupus nephritis confirmed by biopsy with positive autoantibodies is also sufficient for classification [4].

The absence of an SLE diagnostic criteria and the wide variety of clinical manifestations make the diagnosis challenging and by exclusion. It 
requires the appropriate gathering and interpretation of the patient's symptoms, physical signs, and diagnostic tools.

The recommendations for the treatment of SLE are based on an approach combining evidence as well as the opinions of experts in the field. Currently, the only major improvement was to create better protocols based on the use of existing medications, both traditional and biological. There is a newly approved drug for SLE by the name of belimumab. However, it has very limited post-marketing experience as patients with severe renal and central nervous system diseases were excluded from its original studies. It is expected that treatment algorithms will be changed in the future by biologic therapies.

It is important to point out that disease presentations and clinical manifestations vary widely in SLE and that treatment protocols should be thought out carefully and fitted to each patient's unique disease course and needs.

\subsubsection{Management Guidelines for Systemic Lupus Erythematosus}

The goals of treatment of systemic lupus erythematosus are the following:

1. Induction of remission: aiming to rapidly control disease activity for prolonged periods of time.

2. Maintenance therapy: aiming to retain remission or low disease activity and to prevent flares.

3. Adjunctive therapy: aiming to reduce the side effects of drugs employed to control disease activity and to control other SLE-associated conditions.

\subsubsection{General Management Recommendations}

The 2019 European League Against Rheumatism guidelines for the treatment of SLE without renal involvement include [5] the following (Figs. 25.4 and 25.5):
- Hydroxychloroquine (HQ) should be given to all SLE patients with a maximum dose of 5 $\mathrm{mg} / \mathrm{kg} / \mathrm{day}$.

- Ophthalmological examination should be conducted at diagnosis time, after 5 years and then every year to screen for retinal toxicity associated with HQ.

- Oral steroids are to be given in cases of mild disease, while intravenous steroids are to be given in moderate/severe disease.

- It is recommended to keep chronic prednisone use under $7.5 \mathrm{mg}$ daily (or its equivalent) and to stop it whenever possible.

- In patients who are not sufficiently controlled on HQ or those who need further steroidsparing agents, immunosuppressive agents can be added. Methotrexate (MTX) is recommended for mild disease activity. Azathioprine (AZT), calcineurin inhibitors (CNI), and mycophenolate mofetil (MMF) are to be given for moderate disease activity, both as steroidsparing agents and as initial therapies, while belimumab is used for refractory cases. MMF and cyclophosphamide (CYC) are recommended for induction of remission in severe disease activity, while rituximab (RTX) is used when disease response is poor.

- For cutaneous manifestations of SLE, firstline management includes topical steroids and CNI; systemic steroids can also be used, along with HQ. If patients do not respond to initial treatment, further choices should include MTX, retinoids, dapsone, and MMF. Rescue therapy with thalidomide can be considered after failure of all previous lines.

- Acute management of thrombocytopenia due to SLE (Plt < 30,000) includes the use of moderate-/high-dose steroids in combination with either AZT, MMF, or CYC. Intravenous immunoglobulins can also be used in case of poor initial response to steroids. For refractory cases, RTX therapy can be attempted. Last resort choices include thrombopoietin and splenectomy.

- Immunization against seasonal influenza yearly and pneumococcal vaccine (both PCV13 and PPSV23) every 3-5 years is recommended. 

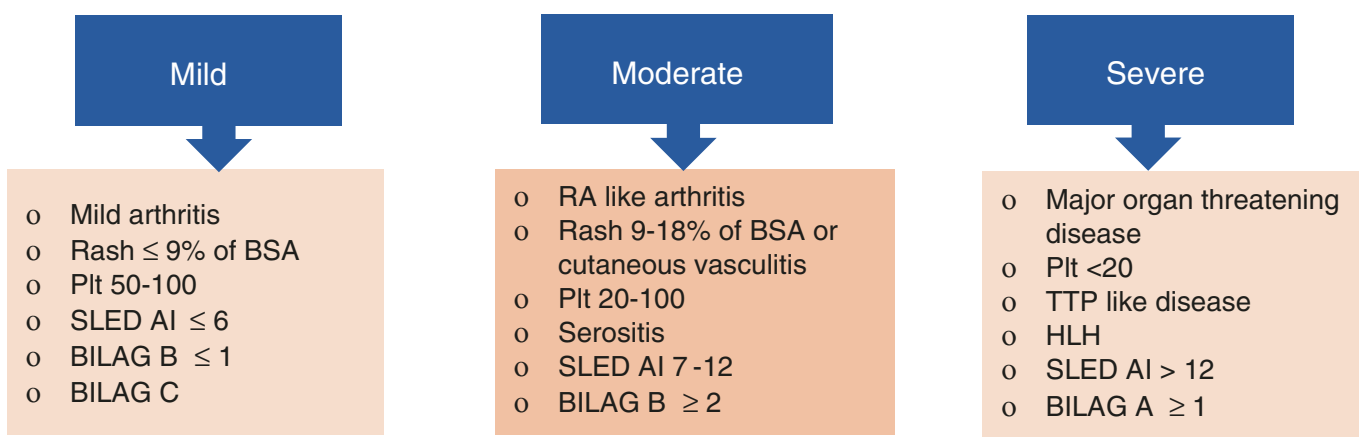

First line

Hydroxychloroquine

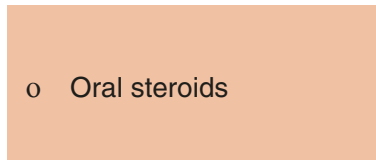

$\begin{array}{ll}\text { o } & \text { Oral or IV steroids } \\ \text { o } & \text { AZA } \\ \text { o } & \text { CNI } \\ \text { o } & \text { MMF }\end{array}$
o Oral or IV steroids
o MMF
o Cyclophosphamide

Did not respond

\title{
Steroids
}

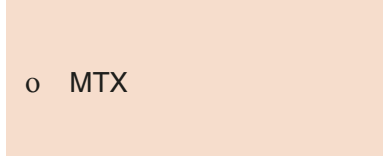

$\begin{array}{ll}\text { o } & \text { Belimumab } \\ \text { o } & \text { CNI } \\ \text { o } & \text { MMF } \\ \text { o } & \text { Steroids }\end{array}$
o CYC
o Rituximab
o Steroids

\begin{abstract}
SLED AI = Systemic Lupus Eythematosusactivity index; BILAG = British Isle Lupus Assessment Group; TTP = thrombotic thrombocytopenic purpura; HLH = hemophagocytic lymphohistocytosis.

Source: The 2016 Update of the EULAR Recommendations for the Management of Systemic Lupus Erythematosus
\end{abstract}

Fig. 25.4 Management of systemic lupus erythematosus without kidney involvement [5]

Recognizing certain clinical manifestations in SLE is crucial as proper treatment should be started promptly to salvage organ function. Lupus nephritis and neuropsychiatric lupus are considered to be the two most serious clinical manifestations of SLE. In the next section, we will introduce the latest published classification criteria and management guidelines for lupus nephritis and neuropsychiatric lupus.

\subsubsection{Lupus Nephritis (LN)}

It is estimated that during the first 10 years of diagnosis, 50-60\% of SLE patients will develop renal disease. Kidney biopsy should be executed in most patients with SLE who have evidence of kidney involvement in order to establish the diagnosis of lupus nephritis and to classify the patient's renal disease according to its histopathology. This will help determine the disease's prognosis and the proper line of therapy that should ensue (see chapter "Renal System and Rheumatology") [6].

The lupus nephritis classification system was developed by the International Society of Nephrology (ISN) in 2003 [7]. This system appears to be associated with increased reproduc- 
Fig. 25.5 Management of specific systemic lupus erythematosus manifestations [5]

\section{Management of Specific Systemic Lupus Erythematosus} Manifestations
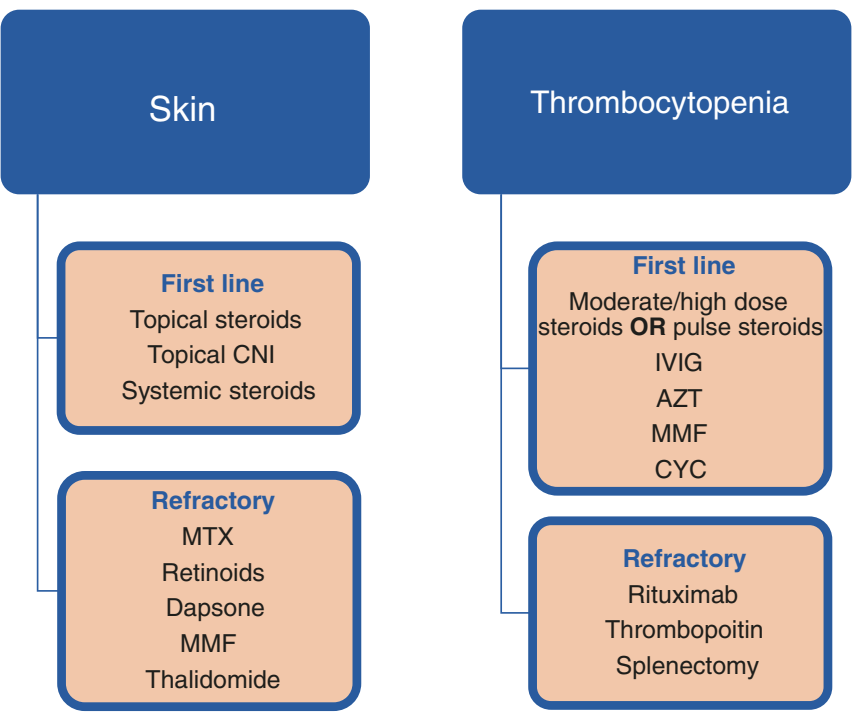

Source: The 2016 Update of the EULAR Recommendations for the Management of Systemic Lupus Erythematosus

ibility compared with the modified 1974 WHO system [8]. The ISN classification system divides glomerular disorders associated with SLE into six different patterns (or classes) [7], each carrying distinct histopathological, clinical, and prognostic characteristics (Fig. 25.6).

The 2012 American College of Rheumatology guidelines for treatment of SLE with renal involvement include the following (Figs. 25.7, 25.8, 25.9, 25.10 and 25.11):

- Renal function monitoring should be done every 3 months in patients deemed at high risk of developing LN, including male patients, juvenile-onset SLE, and seropositivity for anti-C1q antibodies [5].

- The use of either MMF or low-dose CYC along with glucocorticoids is recommended for the induction of remission in Class III and IV LN. Both are equally efficient in controlling the disease with no clear superiority for one over the other [6].
- Higher doses of CYC can be used in LN if there is an increased risk of progressing to end-stage renal disease (decreased glomerular filtration rate, the presence of crescents or fibrinoid necrosis in the kidney biopsy) [5].

- Maintenance therapy with MMF or AZT is recommended for Class III and IV LN [6].

- Use of RTX or CNI along with glucocorticoids is recommended to treat resistant cases of Class III and IV LN that failed traditional therapy [6].

- Class V LN patients are recommended to use MMF only as the induction drug of choice with either MMF or AZT serving as maintenance therapy [6].

- Resistant cases of Class V LN are recommended to use CYC with glucocorticoids [6].

- Evaluation of disease activity every 6 months with modification of treatment options according to the ACR response criteria is recommended [9]. 
Fig. 25.6 The ISN/RPS

2003 classification of lupus nephritis [7].

\section{Lupus Nephritis Classification}

\section{Class I \\ Minimal mesangial LN \\ Class II \\ Mesangial proliferative LN}

\section{Class III Focal LN \\ $<50 \%$ of glomeruli}

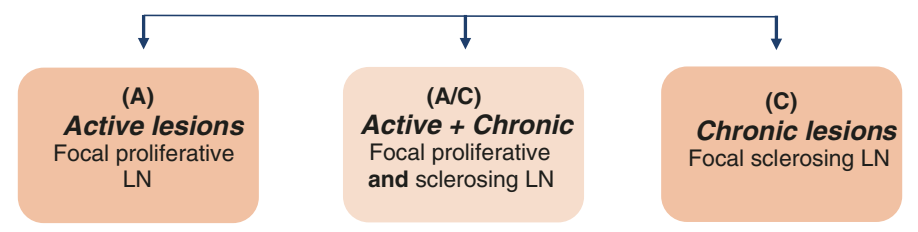

Class IV Diffuse LN

$\geq 50 \%$ of glomeruli

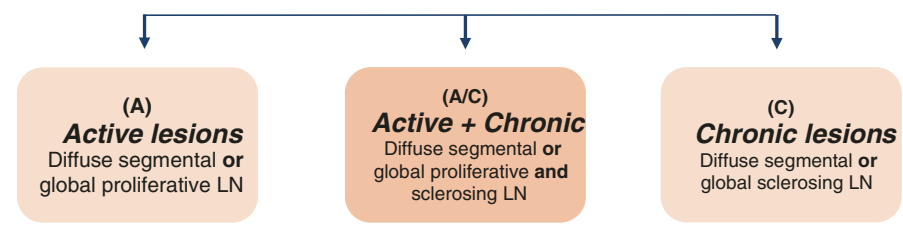

\section{Class V}

Class VI

Membranous LN

Advanced sclerosing LN

\section{SOURCE: THE ISN/RPS 2003 CLASSIFICATION OF LUPUS} NEPHRITIS

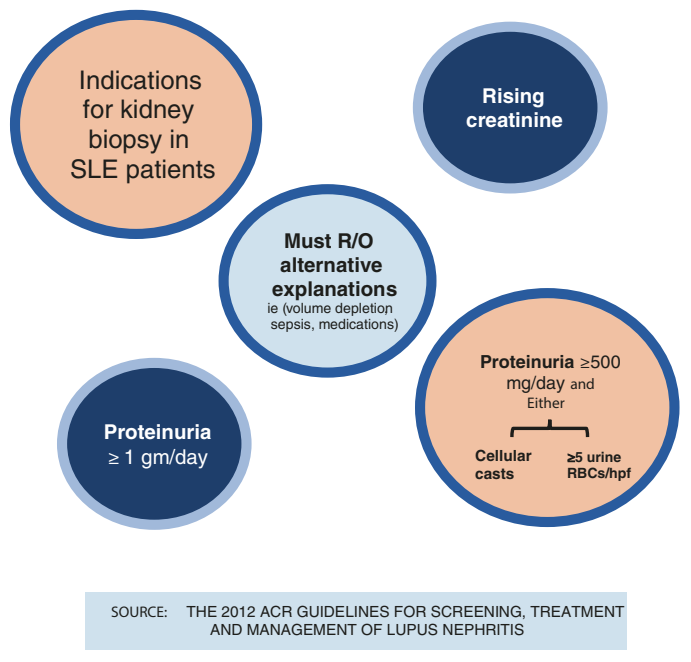

Fig. 25.7 The 2012 American College of Rheumatology indications for kidney biopsy in SLE patients [6]
- Early decrease in urine protein $(\leq 1$ gram at 6 months or $\leq 800 \mathrm{mg}$ at 12 months) carries a favorable long-term prognosis [5].

- There are special considerations for lupus nephritis during pregnancy [6].

- There are special recommendations for adjunctive treatment of lupus nephritis [6].

\subsubsection{Neuropsychiatric Lupus [10]}

SLE is known to affect both the central nervous system (CNS) and peripheral nervous system. It is one of the most confusing manifestations of the disease, and it also carries one of the highest risks of morbidity and mortality. These manifestations may occur prior or during the disease course, with the commonest symptoms including headaches, psychiatric 
Fig. 25.8 The 2012 American College of Rheumatology guidelines for treatment of lupus nephritis [6]

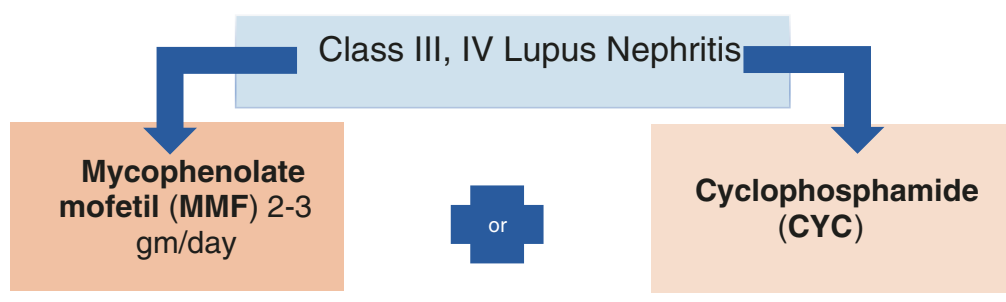

Pulse steroids for 3 days Then

Prednisone $0.5-1 \mathrm{mg} / \mathrm{kg} / \mathrm{day}$ (taper to lowest dose needed for suppression)

\section{Responded}

MMF $1-2 \mathrm{gm} /$ day OR

AZA $2 \mathrm{mg} / \mathrm{kg} /$ day + low dose Prednisone

\section{Did not respond}

Change the regimen MMF

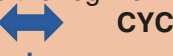

\section{After 6 months}

Pulse steroids

Then

Prednisone

\section{Responded}

Maintenance with MMF 1-2 gm/day OR

AZA $2 \mathrm{mg} / \mathrm{kg} /$ day + Low dose Prednisone
Did not respond

Rituximab

OR

Follow up Calcineurin inhibitors $+$

Steroids

\section{Class VLupus Nephritis}

Responded

Maintenance

MMF 1-2 gm/day

OR

AZA $2 \mathrm{mg} / \mathrm{kg} /$ day

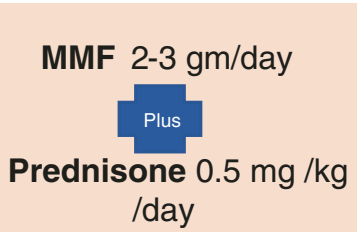

After 6 months

Did not respond

Cyclophosphamide

Plus

Pulse steroids

Then

Prednisone 0.5-1

$\mathrm{mg} / \mathrm{kg} /$ day 


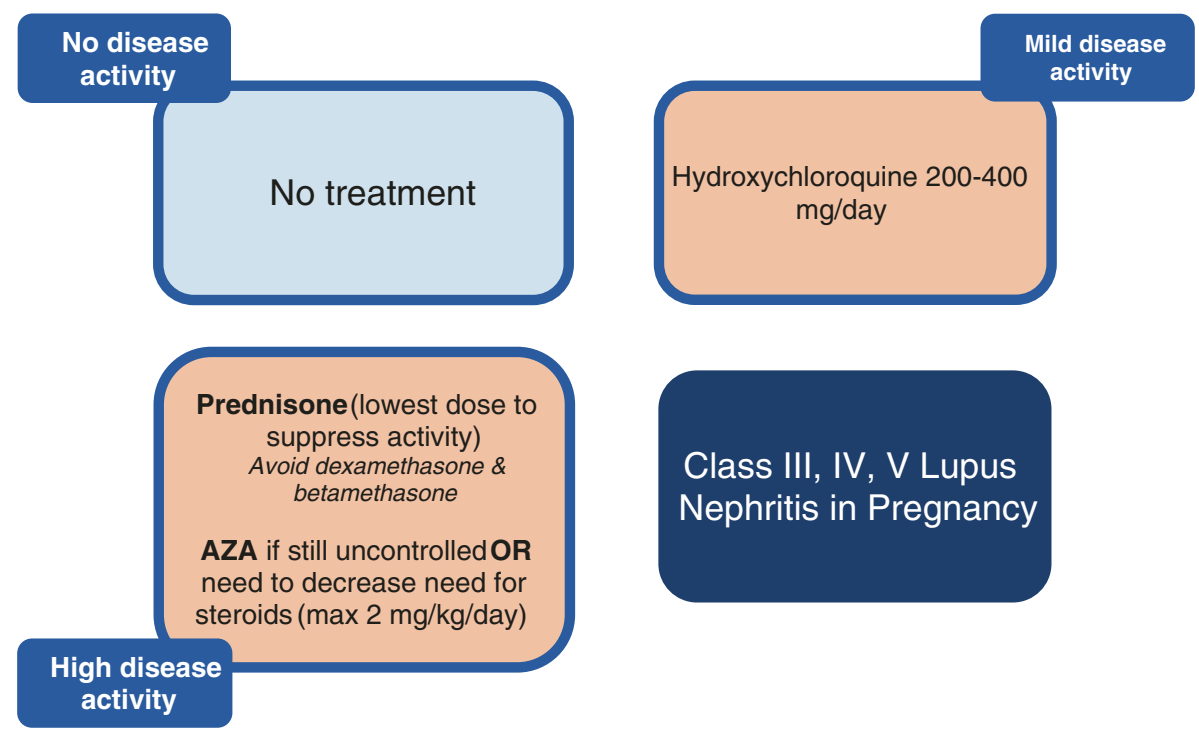

SOURCE: THE 2012 ACR GUIDELINES FOR SCREENING, TREATMENT AND MANAGEMENT OF LUPUS NEPHRITIS

Fig. 25.9 The 2012 American College of Rheumatology guidelines for treatment of class III, IV, and V lupus nephritis in patients who are pregnant [6]

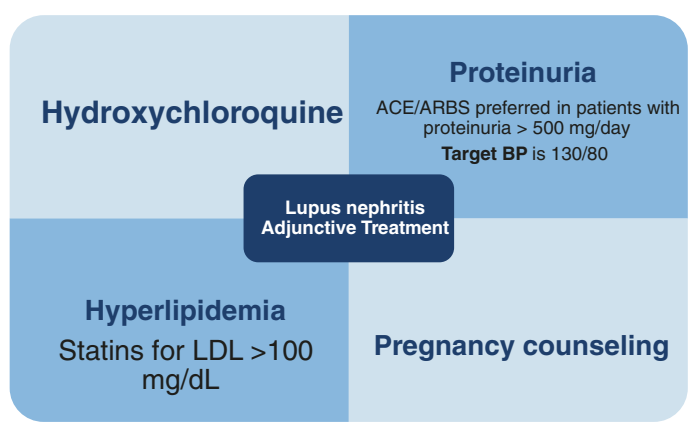

SOURCE: THE 2012 ACR GUIDELINES FOR SCREENING, TREATMENT AND MANAGEMENT OF LUPUS NEPHRITIS

Fig. 25.10 The 2012 American College of Rheumatology guidelines for the adjunctive therapy of lupus nephritis [6]

mood disorders, and cognitive dysfunction. Neuropsychiatric lupus encompasses 19 neurologic and psychiatric syndromes; these were all classified and defined by the ACR. Recommendations for diagnostic testing were also included in these criteria (Figs. 25.12 and 25.13).

The pathogenesis, clinical manifestations, and assessments of neuropsychiatric lupus are very complex which make it difficult to design proper controlled trials; therefore, the treatment is strongly based on physicians' clinical experience. Treatment tends to vary with the manifestation, for example, stroke due to antiphospholipid antibodies is treated with anticoagulants, while cognitive defects may respond to steroids, antidepressants, and/or anxiolytics. There are no randomized clinical trials that have specifically examined these treatments. Principle of management of NPSLE (see Box 25.1, Figs. 25.14 and 25.15) [11]. 
Response Criteria for Lupus Nephritis

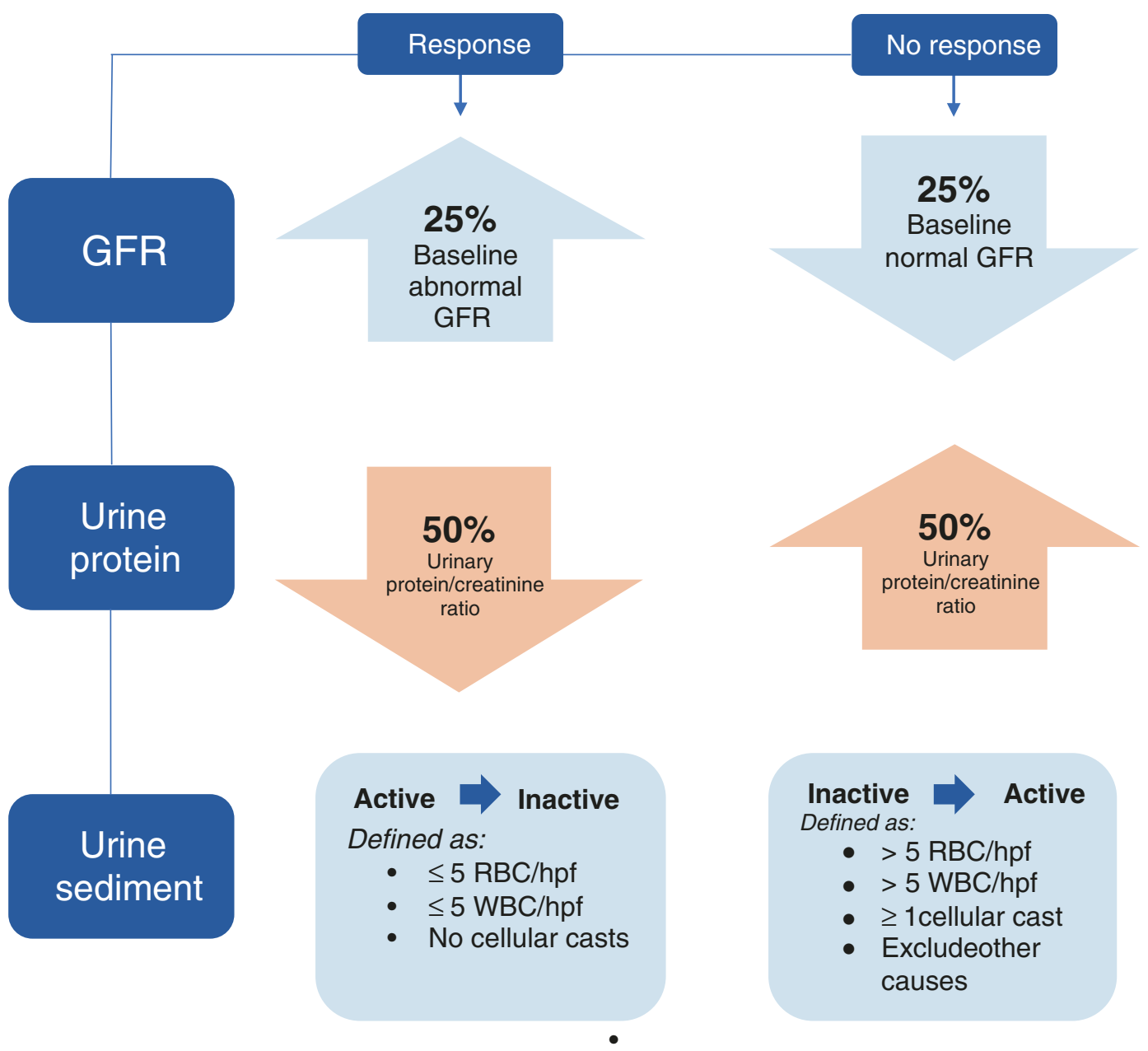

SOURCE:THE 2006 ACR RESPONSE CRITERIA FOR PROLIFERATIVE AND MEMBRANOUS RENAL DISEASE IN SLE CLINICAL TRIALS

Fig. 25.11 The 2006 American College of Rheumatology response criteria for proliferative and membranous renal disease in SLE [9] 


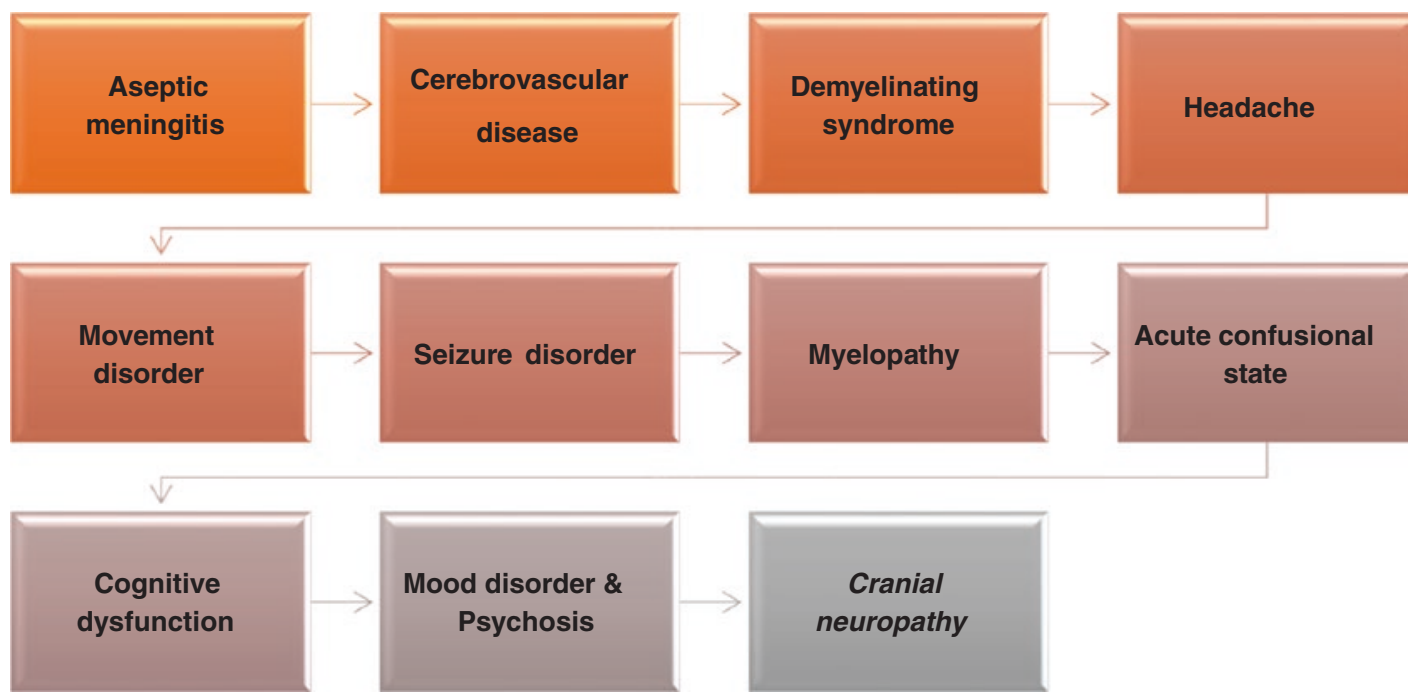

Fig. 25.12 Neuropsychiatric manifestations of systemic lupus erythematosus (Central nervous system)

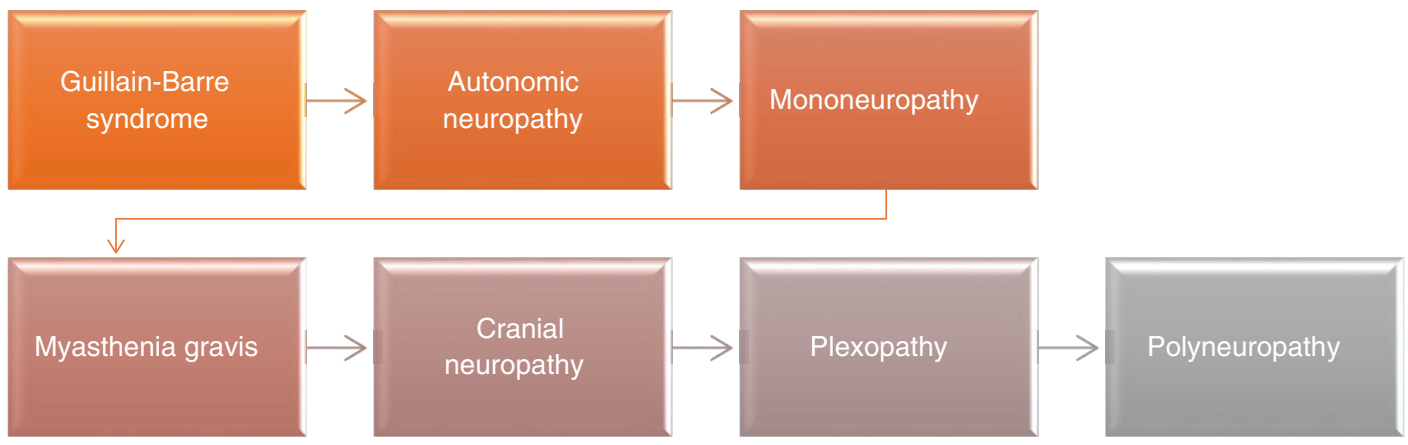

Fig. 25.13 Neuropsychiatric manifestations of systemic lupus erythematosus (peripheral nervous system)

Box 25.1 Principle of management of NPSLE
1-Sympomatic therapy include anticonvulsants, antidepressants and treatment of any aggravating factors

2-Antiplatelet/anticoagulation therapy:Indicated when manifestations are related to antiphospholipid antibodies

3-Glucocorticoids and immunosuppressive therapy are indicated after the exclusion of non-SLE causes if the neuropsychiatric manifestations were felt to reflect an immune inflammatory process (eg: acute confusional state, aseptic meningitis) after exclusionof non-SLE-related causes 


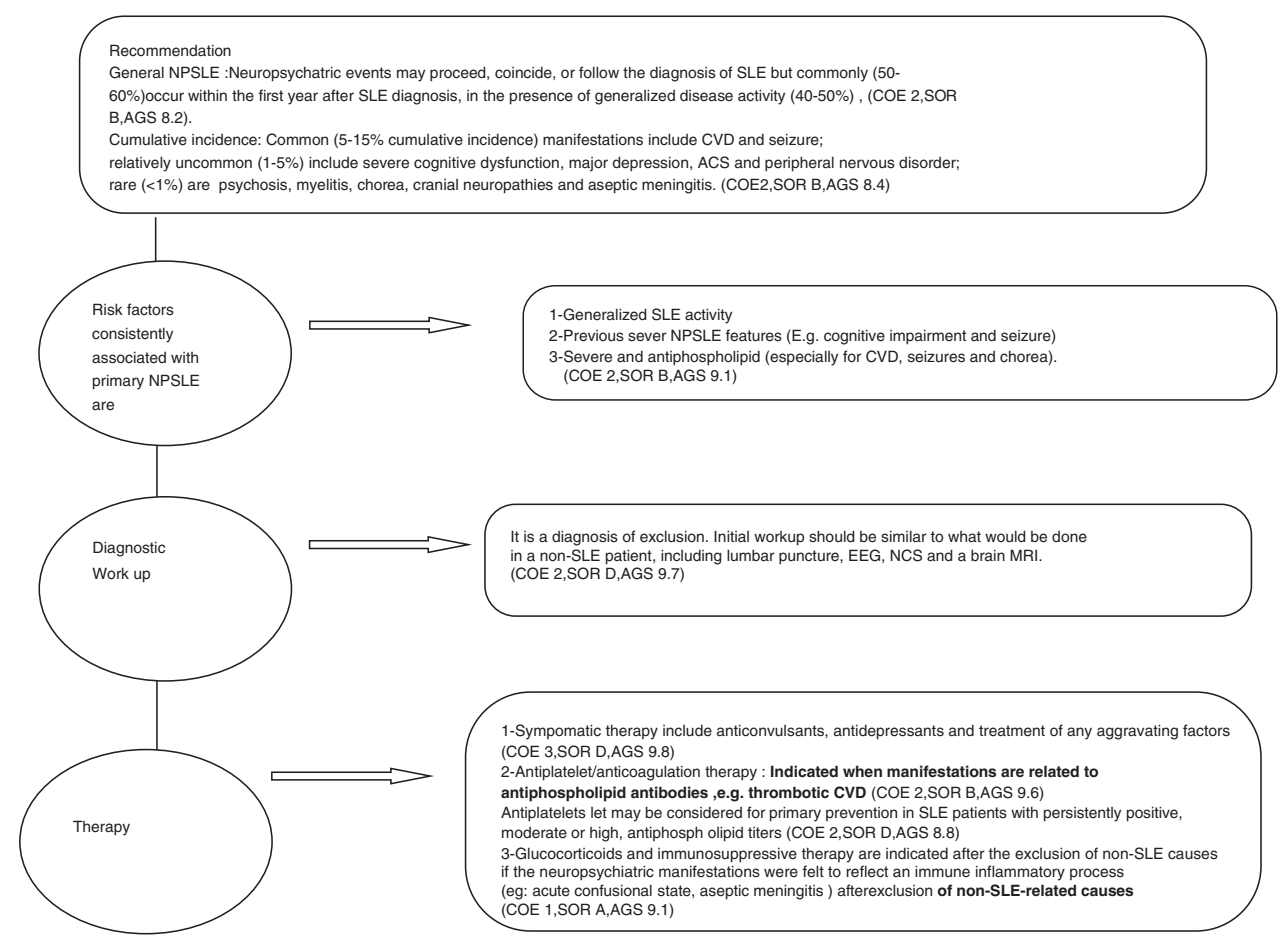

Fig. 25.14 EULAR Recommendation for the management of NPSLE. ACS, acute confusion state; AED, antiepileptic drugs; CNS, central nervous system; CSF, cerebrospinal fluid; CVD, cerebrovascular disease; DWI, diffusion-weighted imaging; FLAIR, fluid-attending

\subsection{Antiphospholipid Syndrome Classification Criteria and Management Guidelines}

\subsubsection{Classification Criteria}

Antiphospholipid syndrome (APS) is an autoimmune disease that mainly causes thrombosis of the patient's arteries and veins and may also lead to poor pregnancy outcomes. The presence of antiphospholipid antibodies (aPL) is associated with this disease. However, these antibodies can also be found in healthy individuals.

The definition has been discussed in several international meetings involving experts from different specialties (rheumatology, obstetrics, neurology, hematology, nephrology, etc.). Classification criteria were proposed in 1998 in Japan. It required positive antibodies testing and at least one clinical manifestation of APL. These criteria were initially intended to be used in inversion recovery sequence; NCS, nerve conduction studies; NPSLE, neuropsychiatric systemic lupus erythematosus; SLE, systemic lupus erythematosus; $\mathrm{COE}$, category of evidence; SOR, strength of recommendation; AGS, agreement score

research settings; however, they were also used by clinicians to decrease the rates of overdiagnosing this disease. Although these criteria helped to classify a homogenous group of patients for research purposes, they had some limitations when used in a clinical setting, as some patients who had clinically evident APL still failed to fulfill these classification criteria. The same group subsequently modified these criteria in 2006, in Sydney. The most significant modifications are outlined below [12]:

(a) Time between two positive antibodies results was lengthened to 12 weeks. This was done to detect persistent positivity.

(b) For the antibody anti-beta-2 glycoproteins, both IgG and IgM antibodies were added to the criteria.

There is a need to further understand the underlying pathogenic mechanisms that cause 
APL. Further studies that develop more specific laboratory techniques to be able to detect those who are most at risk of developing thrombosis and poor pregnancy outcomes are also imperative. These techniques could also help with the recruitment of patients in clinical trials (see Fig. 25.16).

\subsubsection{Management Guidelines}

In the absence of solid guidelines derived from clinical trials, using prophylaxis as a management strategy is still controversial. The treatment of non-obstetric manifestations of APS is mostly the same regardless of the classification of APS (primary vs secondary). Current treatment of APS includes heparin and warfarin. Many patients with coexisting SLE are also treated with hydroxychloroquine, which may have some benefit for patients at risk of thrombosis; this is based on evidence from retrospective studies that suggest the presence of an association between the use of hydroxychloroquine and a reduced risk of thrombosis.

There are special recommendations regarding the treatment of catastrophic APS as well as for the management of APS during pregnancy. More well-designed, prospective research that tackles the management options for APL is required (Fig. 25.17) [13].

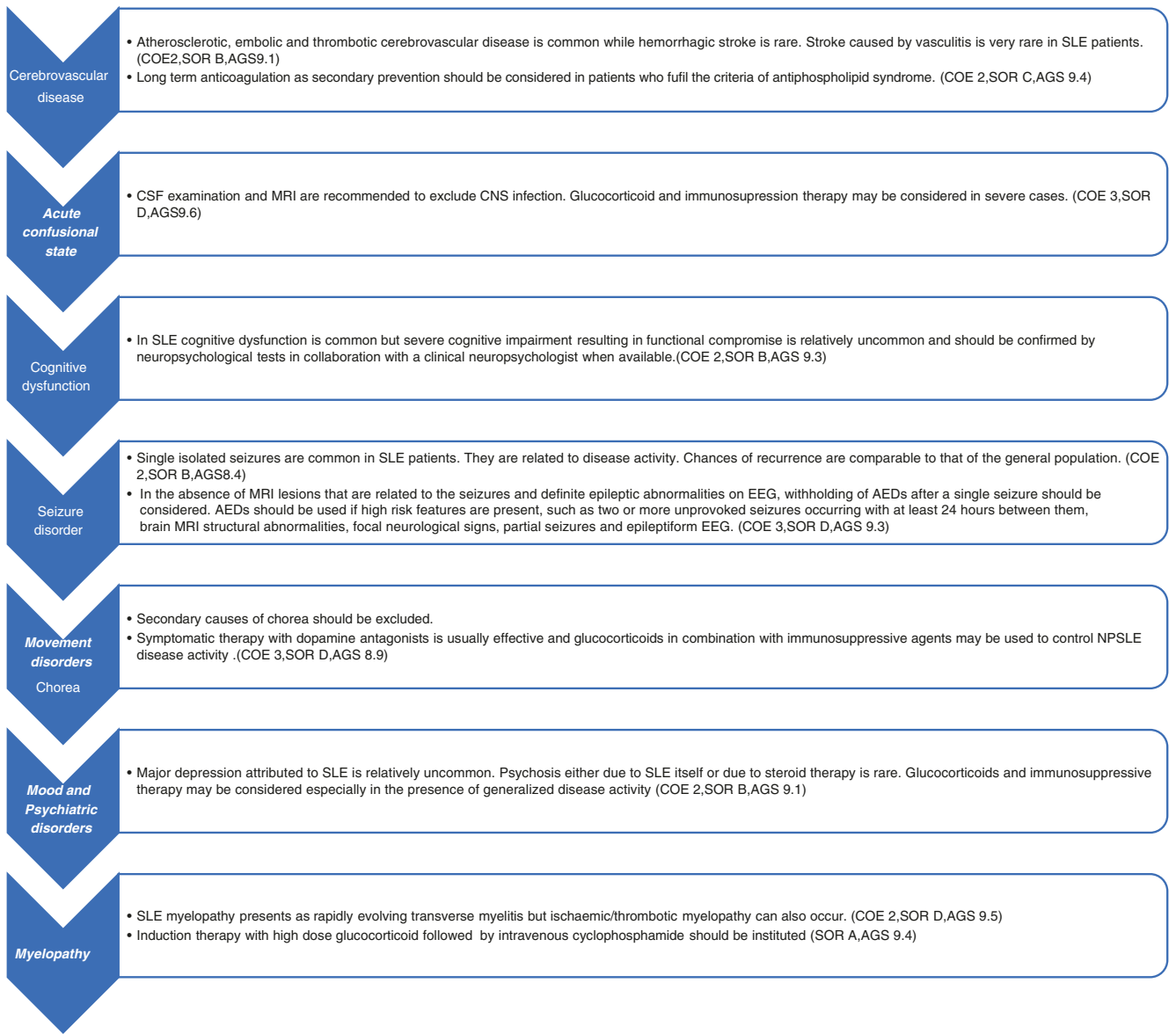

Fig. 25.15 EULAR Recommendation for the Management of Specific NPSLE disorder. ACS, acute confusion state; AED, antiepileptic drugs; CNS, central nervous system; CSF, cerebrospinal fluid; CVD, cerebrovascular disease; DWI, diffusion-weighted imaging;
FLAIR, fluid-attending inversion recovery sequence; NCS, nerve conduction studies; NPSLE, neuropsychiatric systemic lupus erythematosus; SLE, systemic lupus erythematosus; COE, category of evidence; SOR, strength of recommendation; AGS, agreement score 
- Headache alone in an SLE patient requires no further investigation beyond the evaluation, if any, that would have been performed for non-SLE patients. Unless there are high risk feature from the medical history and the physical examination

Headache

\section{Y}

meningitis

- Can be a manifestation of active SLE. Other causes of aseptic meningitis, such as infections, medication, and malignancy, should be excluded

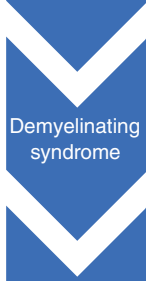

- Can be a clinically isolated syndrome or may overlap with another CNS demyelinating syndrome. However, it should be noted that up to $60 \%$ of NPSLE patients may have oligoclonal bands in their CSF, and evidence suggesting demyelination on imaging is not rare

Cranial

neuropathy

- Optic neuropathy includes inflammatory optic neuritis and ischaemic/thrombotic optic neuropathy. Optic neuritis is commonly bilateral. The diagnostic work up include complete ophthalmological evaluation.

- Glucocorticoids alone or in combination with immunosuppresive therapy should be considered. However failure of therapy is common (COE1,SOR A,AGS9.1)

Fig. 25.15 (continued)

At least one clinical and one laboratory criteria should be met*
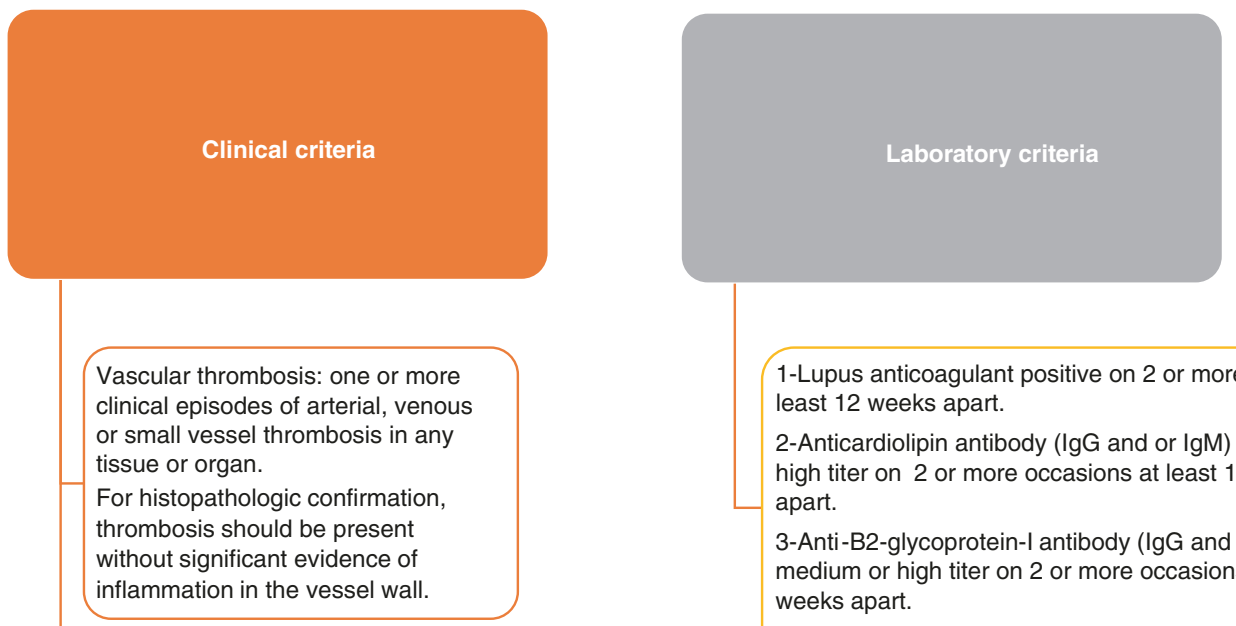

Vascular thrombosis: one or more cinical episodes of arterial, venous tissue or organ.

For histopathologic confirmation, wis should be present inflammation in the vessel wall.

1-Lupus anticoagulant positive on 2 or more occasions at least 12 weeks apart.

2-Anticardiolipin antibody ( $\operatorname{lgG}$ and or $\lg \mathrm{M}$ ) in medium or high titer on 2 or more occasions at least 12 weeks apart.

3-Anti-B2-glycoprotein-I antibody (IgG and or IgM) in medium or high titer on 2 or more occasions at least 12 weeks apart.

-Antibodies measured by a standardized ELISA .

Pregnancy morbidity ${ }^{\star \star *}$ :

1- One or more unexplained deaths of a morphologically normal fetus at or beyond the 10 th week of gestation. Or

2- One or more premature births of a morphologically normal neonate before the 34th week of gestation. Or

3-Three or more unexplained consecutive spontaneous abortions before the 10th week of gestation, with exclusion of maternal anatomic or hormonal abnormalities and paternal or maternal chromosomal abnormalities.

Fig. 25.16 Classification criteria for antiphospholipid syndrome (APS) 


\subsection{Vasculitis Classification Criteria and Management Guidelines}

\subsubsection{Classification Criteria} (Fig. 25.18)

Vasculitides encompasses a group of heterogeneous yet uncommon conditions that can either occur secondary to another disease or arise on its own. Classification criteria of vasculitis generally include several organizing principles like the size of the involved vessel, type of involved vessel (artery, vein, capillary, etc.), underlying pathophysiology (primary vs secondary vasculitis), type of immune damage, and others.

There are no validated criteria for the diagnosis of vasculitis. However, the ACR presented classification criteria in 1990 for seven types of vasculitis. These criteria's main limitation was

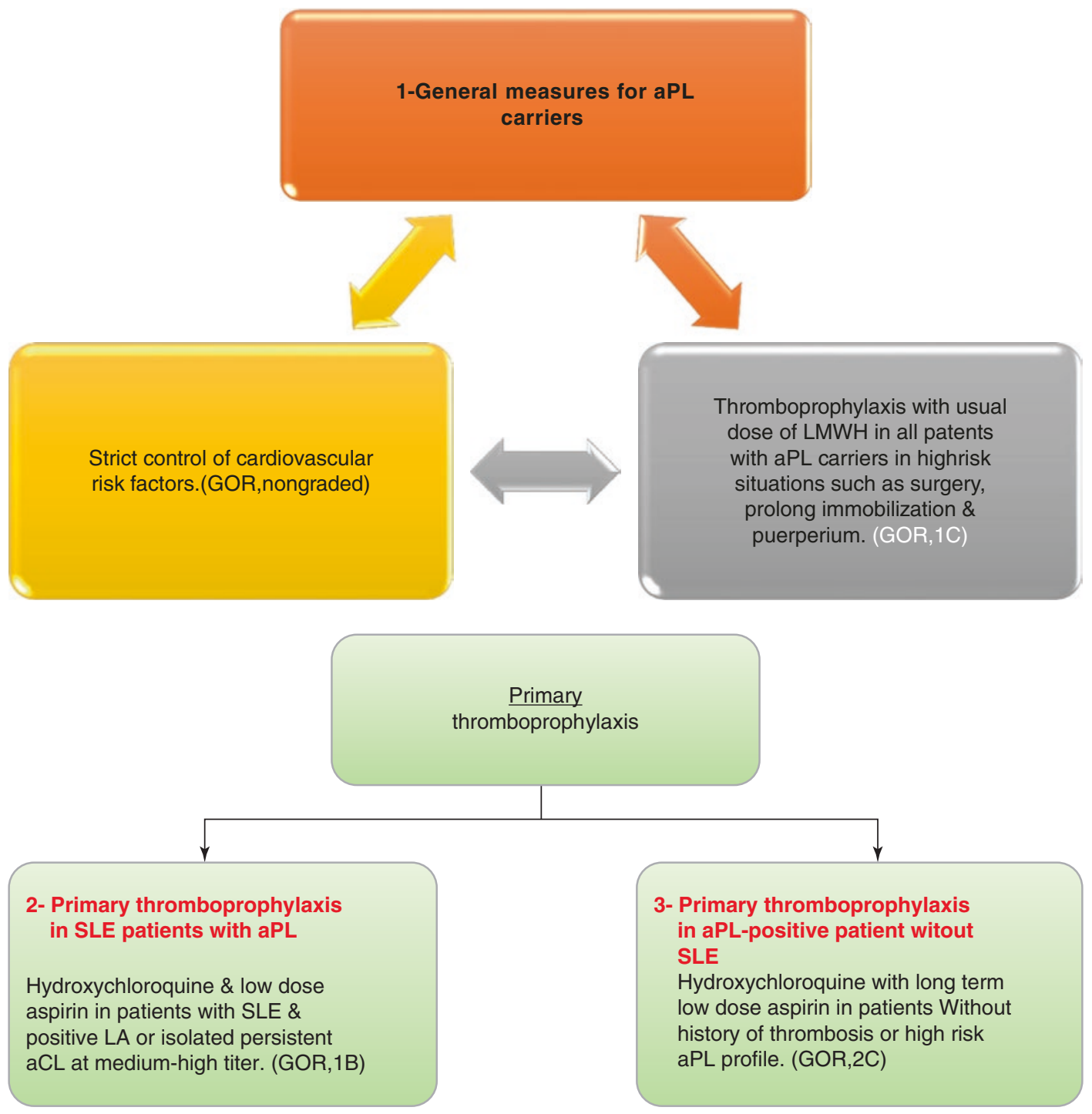

Fig. 25.17 Summary of Management Guideline for Antiphospholipid syndrome(APS) 


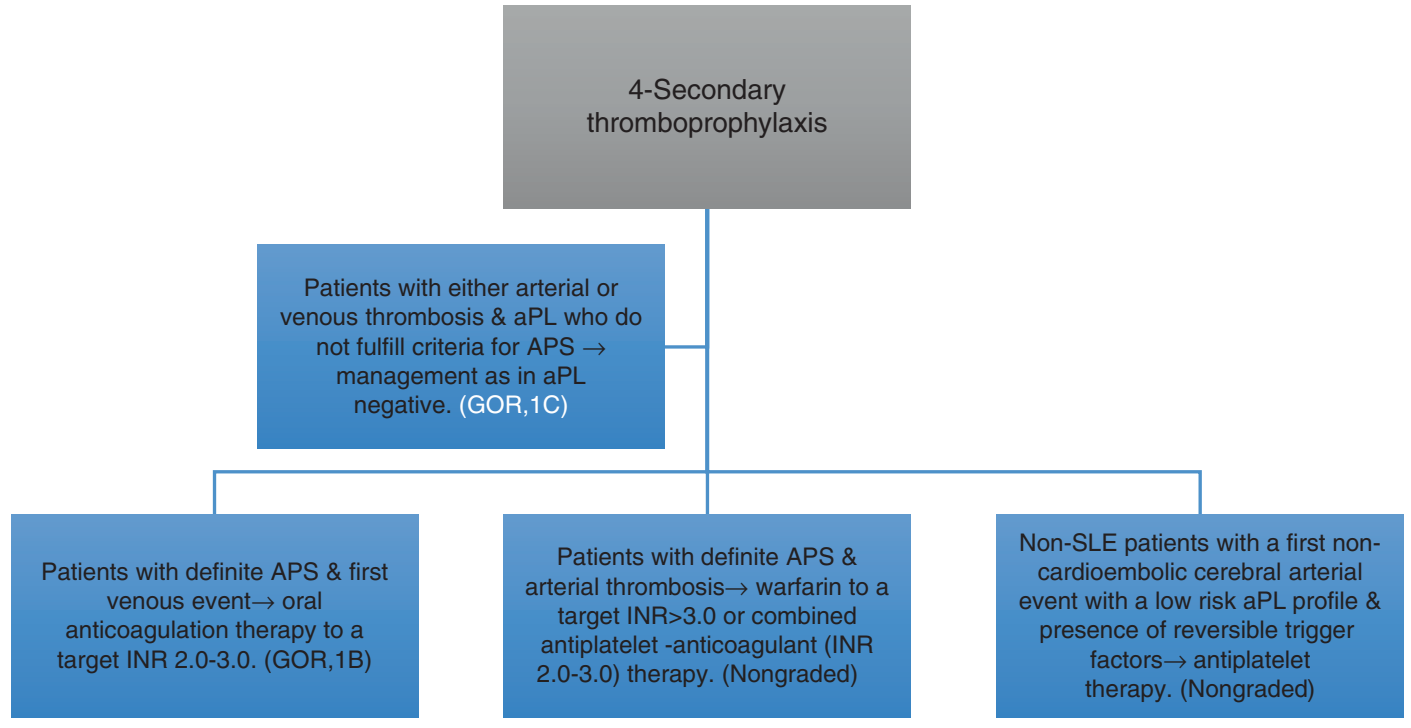

5- Duration of treatment

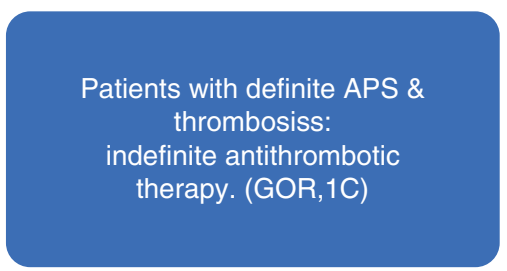

Patients with first venous event, low aPL profile \& known transient precipitating factor $\rightarrow$ 3-6 months of anticoagulation. (Nongraded)

Fig. 25.17 (continued)

that it did not include microscopic polyangiitis or antineutrophil cytoplasmic antibodies (ANCA) [14].

The most widely used nomenclature system is the one introduced in 1994 and revised in 2012 by the Chapel Hill Consensus Conference (CHCC) [15], which included microscopic polyangiitis and replaced disease eponyms with names that were more representative of the disease's underlying pathophysiology. However, unlike the previously mentioned ACR criteria, the CHCC was not meant to be a classification or diagnostic criteria. Although, the ACR and CHCC definitions are widely used, there is no agreement about how it should be applied. Another set of classification criteria is the European Medicines Agency (EMA) criteria, which attempted to produce a consensus method for the application of both the ACR's and the CHCC's definitions of ANCA-associated vasculitis and polyarteritis nodosa in a clinical setting. They developed an algorithm which incorporates the ACR and CHCC definitions with both ANCA and surrogate markers to successfully classify this population of patients [16].

The most notable changes suggested by the 2012 CHCC are:

- Use of the term eosinophilic granulomatosis with polyangiitis (EGPA) instead of ChurgStrauss syndrome.

- Adoption of the term antineutrophil cytoplasmic antibody (ANCA)-associated vasculitis (AAV) instead of three disorders: microscopic 
polyangiitis (MPA), granulomatosis with polyangiitis (GPA) (Wegener's), and EGPA.

- Use of the term immunoglobulin A (IgA) vasculitis instead of Henoch-Schönlein purpura.

- Use of the term cryoglobulinemic vasculitis in place of essential cryoglobulinemic vasculitis.

- Introducing a definition for hypocomplementemic urticarial vasculitis (HUV) (anti-Clq vasculitis).

(See chapter "Vasculitis and Rheumatology.") Physicians should use these criteria with an understanding that they are still a work in progress and that they currently have limited value in clinical practice for diagnosing patients. The diagnosis of vasculitis should also always be confirmed by a tissue biopsy.

\subsubsection{Management Guidelines}

Management of vasculitis relies on the extent of involvement and severity of the vasculitis. For example, a mild drug-induced vasculitis would only require discontinuation of the offending drug. Systemic or more severe forms of vasculitis may require a short or more sustained course of glucocorticoids, a cytotoxic agent, or other medications.

Significant progress has been achieved over the last 30 years in terms of refining the manage-

\section{Vasculitis}

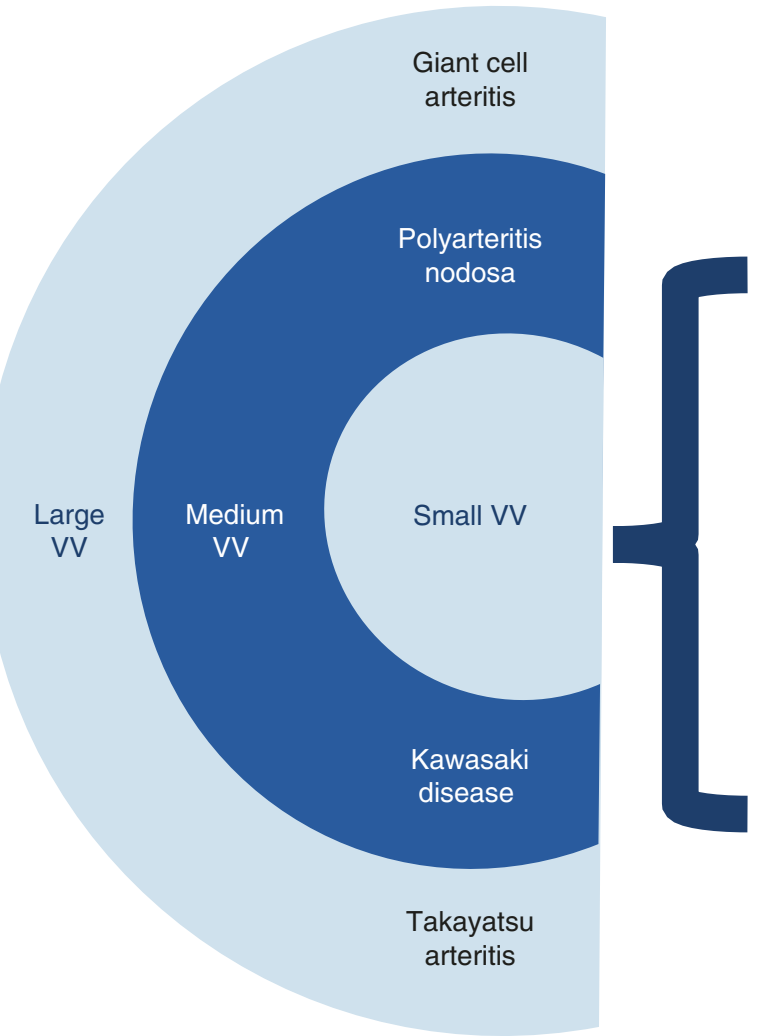

Immune complex

Anti GBM

Cryoglobulinemia IgA vasculitis Hypocomplementemic urticarial vasculitis (anti $\mathrm{C} 1 \mathrm{q}$ vasculitis)

Fig. 25.18 The 2012 Chapel Hill classification criteria (HCCC) of vasculitis [15]

Microscopic polyangiitis Granulomatosis with polyangiitis Eosinophilic granulomatosis with polyangiitis
ANCA associated vasculitis

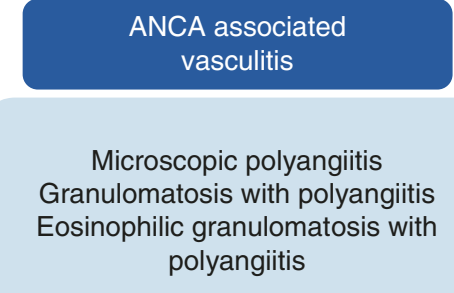


Variable-vessel vasculitis

Behcet syndrome

Cogan's syndrome tis precipitated by an

erlying pathology

Hepatitis Bassociated polyarteritis nodosa

Hepatitis Cassociated cryoglobulinemia

Syphilisaortitis

Malignancy associated vasculitis

Drug inducedANCA vasculitis

Drug induced immune complex vasculitis
Single organ vasculitis

Primary central nervous system vasculitis (CNSV)

Isolated aortitis

Cutaneous leukocystoclastic angiitis

Cutaneous arteritis

Systemic disease associated vasculitis

SLE

RA

Sarcoidosis

SOURCE: THE 2012 INTERNATIONAL CHAPEL HILL

CONSENSUS CONFERENCE ON THE NOMENCLATURE OF

VASCULITIDES

Fig. 25.18 (continued)

ment guidelines of immunosuppressive medications while keeping toxicities at a minimum. These advances have made diseases like ANCAassociated vasculitis (AAV) treatable and less fatal. Further advances are needed as there are still a proportion of patients that are going to develop symptoms that are refractory to all available therapies. Half of this patient population will also develop a relapse within 5 years of diagnosis, and toxicity from treatments given is still a significant contributor to mortality and chronic disability.

The introduction of biomarkers has also made it possible to determine disease activity and estimate risks of relapse. However, the key to adequately managing these patients should be by tailoring their immunosuppressive regimens to their individual needs.

The 2016 European League Against Rheumatism (EULAR)/European Renal Association (ERA)- European Dialysis and Transplant Association (EDTA) recommendations for the management of ANCA-associated vasculitis include the following [17] (Fig. 25.19): - It is recommended to do a biopsy for all patients who are suspected to have vasculitis, or patients who are suspected to have relapsing vasculitis.

- Induction therapy of non-organ-threatening vasculitis should include a combination of 


\section{Management of ANCA-Associated Vasculitis}
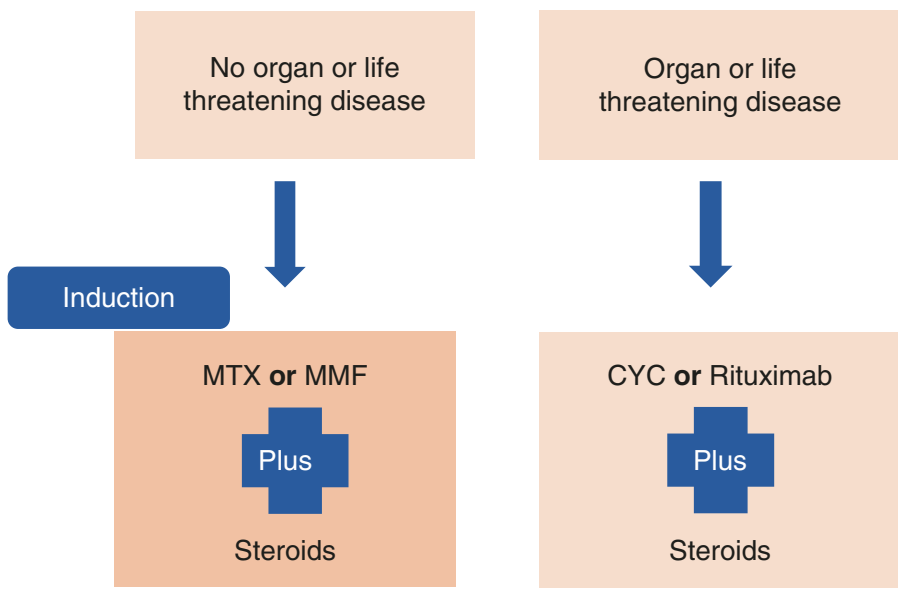
Renal failure with creatinine $>500$ OR
Alveolar hemorrhage
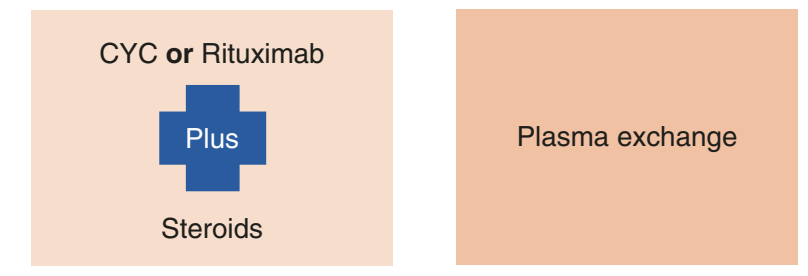

\section{Follow up}

\section{Maintenance}
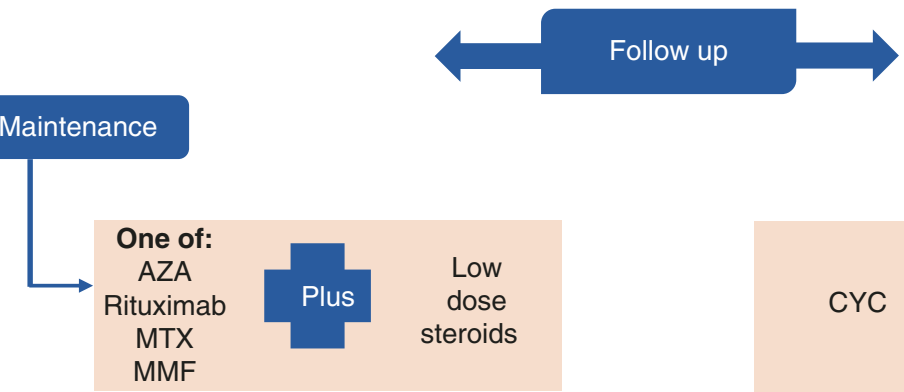

Refractory

SOURCE: THE 2016 EULAR/ERA-EDTA RECOMMENDATIONS FOR THE MANAGEMENT OF ANCA-ASSOCIATED VASCULITIS

Fig. 25.19 The 2016 Eular/ERA-EDTA recommendations for the management of anca-associated vasculitis [17].

glucocorticoids and either methotrexate or mycophenolate mofetil.

- Induction therapy of organ-threatening vasculitis should include a combination of glucocorticoids and either cyclophosphamide or rituximab.

- Relapsing disease with organ-threatening vasculitis should be treated the same as new-onset organ-threatening vasculitis.

- Rapidly progressive glomerulonephritis, both new in onset and relapsing, should be treated with plasma exchange.
- Severe diffuse alveolar hemorrhage should also be treated with plasma exchange.

- For disease maintenance, it is recommended to use a combination of glucocorticoids with either azathioprine, methotrexate, mycophenolate mofetil, or rituximab. This treatment should be continued for a minimum of 24 months.

- For refractory cases, it is recommended to switch from cyclophosphamide to rituximab or from rituximab to cyclophosphamide. 


\subsubsection{Classification Criteria [18]}

Polymyalgia rheumatica (PMR) is an inflammatory disorder that is mainly characterized by neck, shoulder, and hip girdle pain and morning stiffness. An association between PMR and giant cell arteritis (GCA) was found, which may represent a shared underlying pathogenic process.

In April of 2012, the ACR and EULAR convened and proposed PMR classification criteria that were designed to define its most important manifestations. [18].

Scoring-based criteria were made that outlined the following components:

- The presence of morning stiffness for more than 45 minutes ( 2 points).

- Pain in the hips with limited range of motion (1 point).

- The absence of rheumatoid factor and/or anticitrullinated protein antibody ( 2 points).

- The absence of pain in the peripheral joints (1 point).

The interpretation of the PMR scoring algorithm after ruling out alternative conditions:

- The scoring scale is $0-6$ (without ultrasound) and $0-8$ (with ultrasound).

- A score of $\geq 4$ (without ultrasound) or $\geq$ 5(with ultrasound) is suggestive of PMR.

- A score of $>5$ increases the sensitivity to $66 \%$ and specificity to $81 \%$.
- Patients with a score of $<4$ make them less likely to have PMR.

Ultrasounds are the imaging of choice for PMR, as they are a great tool to discern between PMR and other non- inflammatory conditions. They also increase the specificity of diagnosis. These criteria need to be validated by other cohort studies as it is important to distinguish PMR from other conditions (see Table 25.1).

There are no clear guidelines for the treatment of PMR. The role of the early introduction of DMARDs in PMR is not entirely known. Currently, corticosteroids are the mainstay of treatment for PMR, although some randomized controlled trials have studied the use of immunosuppressant therapy. New trials are also studying the use of biologics with PMR (see Table 25.2) [19].

\subsection{Spondyloarthritis Classification Criteria and Management Guidelines}

\subsubsection{Classification Criteria}

(Figs. 25.20, 25.21 and 25.22) (Table 25.3)

Spondyloarthritis $(\mathrm{SpA})$ is an umbrella term that encompasses the following interconnected diseases: ankylosing spondylitis (AS), psoriatic arthritis (PsA), reactive arthritis, enteropathic-related spondylitis and arthritis, and

Table 25.1 Summary of EULAR/ACR 2012 classification criteria for Polymyalgia Rheumatica (PMR)

\begin{tabular}{l|l|l}
\hline Criteria & $\begin{array}{l}\text { Points without } \\
\text { US }(0-6)\end{array}$ & Points with US (0-8) \\
\hline Morming stifness duration $>45 \mathrm{~min}$ & Two points & Two points \\
\hline Hip pain or limited range of motion & One point & One point \\
\hline Absence of RF or ACPA & Two point & Two points \\
\hline Absence of other joint involvement & One point & One point \\
\hline $\begin{array}{l}\text { At least one shoulder with subdeltoid bursitis and/or biceps } \\
\text { tenosynovitis and/or glenohumeral synovitis (either posterior or axillary) } \\
\text { and at least one hip with synovitis and/or trochanteric bursitis }\end{array}$ & Not applicable & One point \\
\hline $\begin{array}{l}\text { Both shoulders with subdetoid burstis, biceps tenosynovitis or } \\
\text { glenohumeral synovitis }\end{array}$ & Not applicable & One point \\
\hline
\end{tabular}

${ }^{a}$ A score of 4 or more is categorized as PMR in the algorithm without US and a score of 5 or more is categorized as PMR in the algorithm with US 
Table 25.2 Summary of EULAR/ACR 2015 recommendation of management for Polymyalgia Rheumatica (PMR)

\begin{tabular}{l|l}
\hline \multicolumn{2}{l}{ Management of Polymyalgia Rheumatica } \\
\hline $\begin{array}{l}\text { Initial glucocorticoid therapy: Prednisone } 15 \mathrm{mg} \text { daily with clinical response in } 1 \text { week and resolution of acute } \\
\text { phase reactant in 3-4 weeks }\end{array}$ \\
\hline If no response & Dose may be increased by $5 \mathrm{mg}$ day increments each week up to $30 \mathrm{mg} /$ day \\
\hline Follow up in & $\begin{array}{l}\text { Gradual steroid tapering. } \\
\text { Once the dose has fallen to } 10 \mathrm{mg} \text { day, reduce the dose no faster than } 1 \mathrm{mg} \text { per month }\end{array}$ \\
\hline Relapse & $\begin{array}{l}\text { - In patients who relapse while on glucocorticoids } \rightarrow \text { increase glucocorticoid dose to lowest } \\
\text { effective dose. } \\
\text { Relapse following discontinuation of glucocorticoids } \rightarrow \text { resumption of glucocorticoids at the } \\
\text { original dose at which control was achieved. } \\
\text { In patients who relapse several times } \rightarrow \text { interval between dose reductions should be increased } \\
\text { to every two or three months. }\end{array}$ \\
\hline
\end{tabular}

Fig. 25.20 ASAS classification criteria for axial spondyloarthritis $(\mathrm{SpA})$

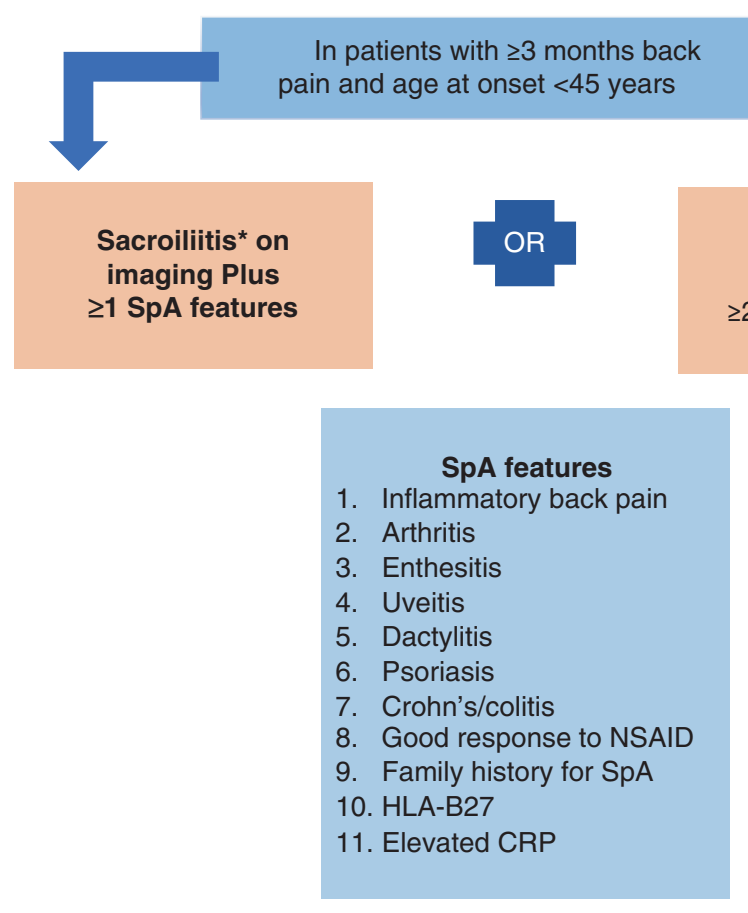

undifferentiated SpA. There are many proposed classification criteria for SpA; however, they are more geared to be used in research contexts rather than clinical settings.

The most recent classification criteria developed by the Assessment of SpondyloArthritis international Society (ASAS) have determined that MRIs are the imaging modality of choice for the detection of axial and sacroiliac affection, as they are more sensitive than radiographs, especially for early disease. These changes were not mentioned in the modified New York criteria, the European Spondyloarthropathy Study Group criteria, and the Amor criteria for AS [20].
The ASAS group also proposed a definition for inflammatory back pain and criteria for classifying axial and peripheral spondyloarthritis. These criteria were designed to provide a diagnosis for patients in the early phases of their disease. The above mentioned advances were made to aid research into the use of biologic agents in early disease. ASAS also defined non-radiographic axial spondyloarthritis (nr-axSpA); this entity shares the same underlying genetic factors, disease course, and prognosis as the radiographic variant; however, it differs in its absence from detection by plain radiographs as well as a lesser degree of ossification and inflammation found both clinically and on MRIs. 


\subsubsection{Management Guidelines}

Diagnosis of SpA is often delayed with many patients not receiving the appropriate treatment. Biological agents were found to halt the disease's progression and improve its prognosis. Treatment, however, should be tailored to each patient's specific needs [21].

ASAS-EULAR Recommendations for Management of SpA (2016 Update) [22] (Figs. 25.23 and 25.24).

The management of patients with AS should be specifically individualized for each patient according to the disease's severity and activity as well as the patient's general condition, function, disability, wishes, and expectations.

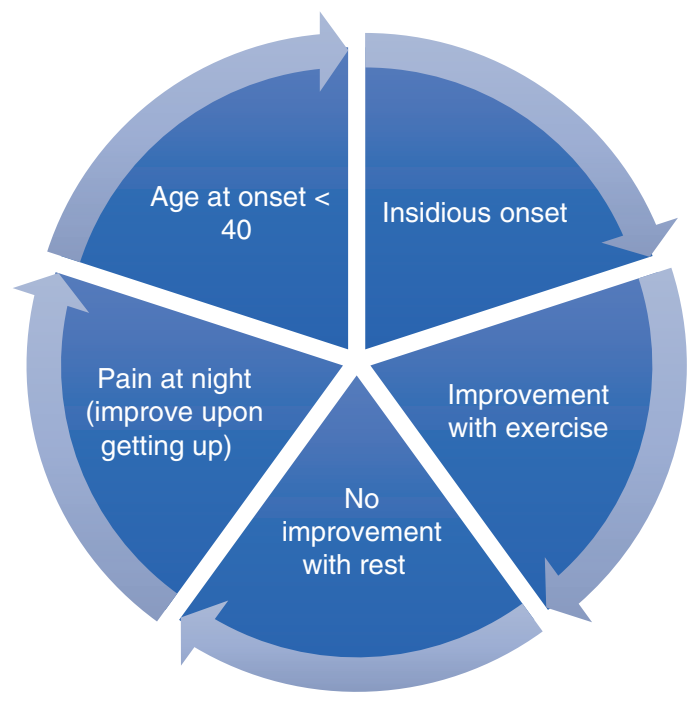

Fig. 25.21 Inflammatory back pain assessment ASAS expert criteria)
Monitoring of the patient's disease includes assessment by history, physical examination, laboratory investigation, and imaging modalities. Management options are then tailored based on this assessment and assigned to either nonpharmacologic, pharmacologic, or surgical approaches.

Nonpharmacologic strategy:

Education, regular exercise, and physiotherapy should be considered.

Pharmacologic strategy:

- Anti-inflammatory drugs are the first-line agents in patients complaining of pain and/or stiffness.

- Pain killers like paracetamol and opioids.

- Local steroid injections can be used if the patient is still in pain despite using antiinflammatory medications.

- DMARDs can be used in extra-axial inflammatory joint pain.

- Anti-tumor necrosis factor (anti-TNF): for patients with axial disease, the use of the combination of DMARDs and anti-TNF is not necessary.

- Interleukin 17 and interleukin 12/23 inhibitors: they have been introduced in the recent ASAS-EULAR guidelines based on recent randomized controlled trials that show their efficacy in SpA.

Surgical strategy:

Total hip replacement and spinal surgery should be contemplated in patients with pain or disability that are refractory to treatment.
Fig. 25.22 ASAS

classification criteria for peripheral spondyloarthritis (SpA)

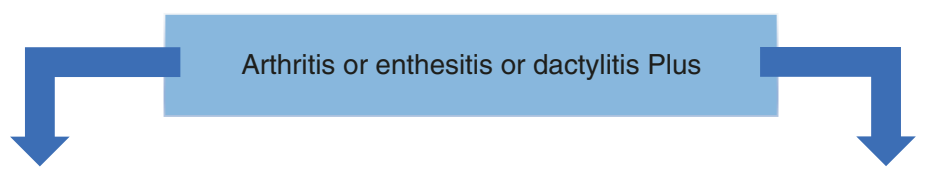

$\geq 1$ SpA features

1. Uveitis

2. Psoriasis

3. Crohn's/colitis

4. Preceding infection

5. HLA-B27

6. Sacroiliits*on imaging $\geq 2$ other SpA features

7. Arthritis

8. Enthesitis

9. Dactylitis

10. IBP (ever)

11. Family history for $\mathrm{SpA}$ 
Table 25.3 Definition of SpA features in the ASAS classification criteria for peripheral SpA

\begin{tabular}{|c|c|}
\hline SpA feature & Definition \\
\hline \multicolumn{2}{|r|}{ Entry criteria: } \\
\hline Arthritis & $\begin{array}{l}\text { Current peripheral arthritis compatible with SpA (usually asymmetric and/or predominant } \\
\text { involvement of the lower limb), diagnosed clinically by a physician }\end{array}$ \\
\hline Enthesitis & Current enthesitis, diagnosed clinically by a doctor \\
\hline Dactylitis & Current dactylitis, diagnosed clinically by a doctor \\
\hline \multicolumn{2}{|r|}{ Additional SpA features: } \\
\hline $\begin{array}{l}\text { IBP in the } \\
\text { past }^{\mathrm{a}}\end{array}$ & IBP in the past according to the rheumatologist's judgement \\
\hline Arthritis & $\begin{array}{l}\text { Past or present peripheral arthritis compatible with SpA (usually asymmetric and/or predominant } \\
\text { involvement of the lower limb), diagnosed clinically by a physician }\end{array}$ \\
\hline Enthesis $^{\mathrm{b}}$ & Enthesitis: Past or present spontaneous pain or tenderness on examination of an enthesis \\
\hline Uveitis & Past or present uveitis anterior, confi rmed by an ophthalmologist \\
\hline Dactylitis & Past or present dactylitis, diagnosed by a physician \\
\hline Psoriasis & Past or present psoriasis, diagnosed by a physician \\
\hline IBD & Past or present Crohn's disease or ulcerative colitis diagnosed by a physician \\
\hline $\begin{array}{l}\text { Preceding } \\
\text { infection }\end{array}$ & Urethritis/cervicitis or diarrhea within 1 month before the onset of arhthritis, enthesitis or dactylitis \\
\hline $\begin{array}{l}\text { Family } \\
\text { history for } \\
\text { SpA }\end{array}$ & $\begin{array}{l}\text { Presence in first-degree (mother, father, sisters, brothers, children) or second-degree } \\
\text { (maternal and paternal grandparents, aunts, uncles, nieces, and nephews) relatives } \\
\text { of any of the following: ankylosing spondylitis, psoriasis, acute uveitis, } \\
\text { reactive arthritis or IBD }\end{array}$ \\
\hline HLA-B27 & Positive testing according to standard laboratory techniques \\
\hline $\begin{array}{l}\text { sacroiliitis by } \\
\text { imaging }\end{array}$ & $\begin{array}{l}\text { Bilateral grade } 2-4 \text { or unilateral grade } 3-4 \text { sacroiliitis on plain radiographs, according } \\
\text { to the modified New York criteria, or active sacroiliitis on MRI according to the } \\
\text { ASAS consensus definition }\end{array}$ \\
\hline
\end{tabular}

ASAS, Assessment of SpondyloArthritis International Society; HLA-B27, human leucocyte antigen B27; IBD, inflammatory bowel disease; IBP, inflammatory back pain; SpA, spondyloarthritis.

${ }^{a}$ Here, only IBP in the past is considered. In patients with current IBP (and concomitant peripheral manifestations), the ASAS classifi cation criteria for axial SpA should be applied

${ }^{b}$ Any site of enthesitis can be affected whereas in the ASAS classification criteria for axial SpA only enthesitis of the head is considered

Fig. 25.23 ASAS-

EULAR

recommendations for the

management of axial

and peripheral

spondyloarthritis)

ASAS-EULAR

recommendations for the treatment of patients with axSpA with bDMARDs.

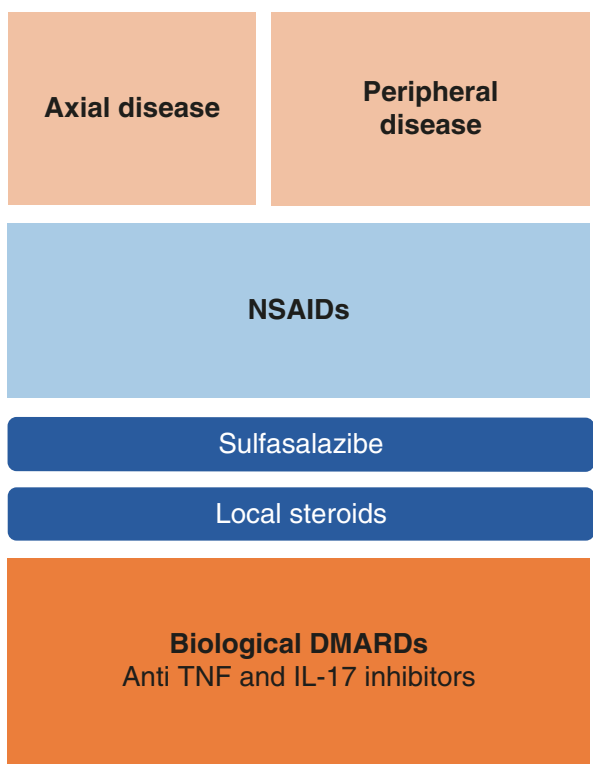

(All patients) Education, exercise, physical therapy, rehabilitation, patient associations, self-help groups, smoking cessation

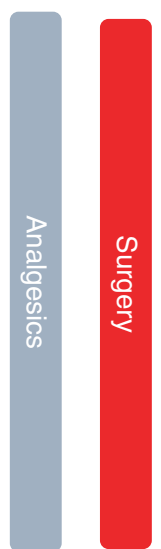


Fig. 25.24 ASAS-

EULAR

recommendations for the treatment of patients with axSpA with bDMARDs)
Diagnosis of axial SpA by a rheumatologist

Plus

High CRP and/or positive MRI and/or radiographic sacroiliitis*

Plus

Failure of standard treatment: all patients

1. at least 2 NSAIDs over 4 weeks (in total)

patients with predominant peripheral manifestations

1. one local steroid injection if appropriate

2. normally a therapeutic trial of sulfasalazine

Plus

High disease activity: ASDAS $\geq 2.1$ or BASDAI $\geq 4$

Plus

Positive rheumatologist's opinion

Table 25.4 Classification Criteria for Psoriatic Arthritis (CASPAR)

Classification criteria for psoriatic arthritis (CASPAR)

Inflammatory articular disease (joint, spine or entheseal) with $\geq 3$ of the following:

\begin{tabular}{|c|c|c|}
\hline \multirow[t]{3}{*}{$\begin{array}{l}\text { 1. Evidence of psoriasis: } \\
\text { (one of } a, b, c \text { ) }\end{array}$} & $\begin{array}{l}\text { a. Current } \\
\text { psoriasis }\end{array}$ & $\begin{array}{l}\text { Psoriatic skin or scalp disease present today as judged } \\
\text { by a rheumatologist or dermatologist }\end{array}$ \\
\hline & $\begin{array}{l}\text { b. Personal } \\
\text { history of } \\
\text { psoriasis }\end{array}$ & $\begin{array}{l}\text { A history of psoriasis that may be obtained from } \\
\text { patient, family doctor, dermatologist, rheumatologist, } \\
\text { or other qualified health-care provider }\end{array}$ \\
\hline & $\begin{array}{l}\text { c. Family } \\
\text { history }\end{array}$ & $\begin{array}{l}\text { A history of psoriasis in a first or second degree relative } \\
\text { according to the patient's reporting }\end{array}$ \\
\hline 2. Psoriatic nail dystrophy & \multicolumn{2}{|c|}{$\begin{array}{l}\text { Typical psoriatic nail dystrophy including onycholysis, } \\
\text { pitting and hyperkeratosis observed on current physical } \\
\text { examination }\end{array}$} \\
\hline $\begin{array}{l}\text { 3. A negative rheumatoid } \\
\text { factor }\end{array}$ & \multicolumn{2}{|c|}{$\begin{array}{l}\text { By any method except latex but preferably by ELISA or } \\
\text { nephelometry, according to the local laboratory } \\
\text { reference range }\end{array}$} \\
\hline 4. Dactylitis (a or b) & \multicolumn{2}{|c|}{$\begin{array}{l}\text { a. Current swelling of entire digit } \\
\text { b. A history of dactylitis recorded by a rhematologist }\end{array}$} \\
\hline $\begin{array}{l}\text { 5. Radiological evidence } \\
\text { of juxta-articular new } \\
\text { bone formation }\end{array}$ & \multicolumn{2}{|c|}{$\begin{array}{l}\text { III-defined ossification near joint margins (but excluding osteophyte formation) on } \\
\text { plain X-rays of hands or feet }\end{array}$} \\
\hline
\end{tabular}

\subsection{Psoriatic Arthritis Classification Criteria and Management Guidelines (Table 25.4) (Fig. 25.25)}

\subsubsection{Classification Criteria}

Psoriatic arthritis (PsA) is an inflammatory joint disease with heterogeneous presentation pat- terns representing different clinical subcategories. Many classification criteria have been put forth but they were not used widely and have not been validated. The presence of these different presentation patterns has made it difficult to propose and validate classification criteria for the diagnosis of PsA, especially with disease patterns like seronegative polyarthritis and psoriasis. 


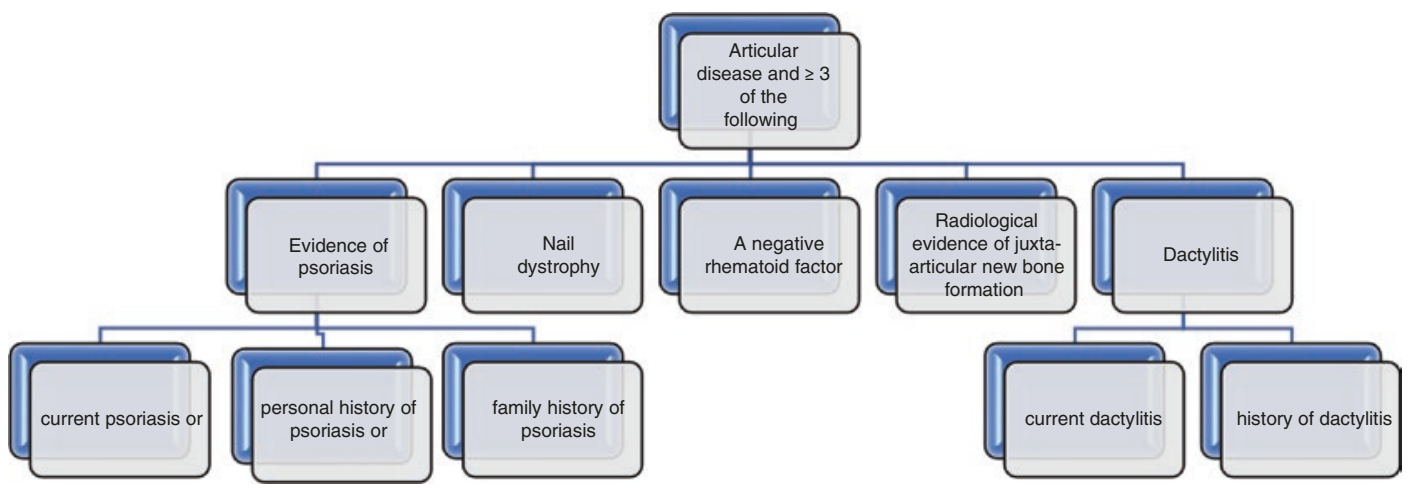

Fig. 25.25 Summary of the Classification Criteria of Psoriatic Arthritis

Older criteria, such as the Moll and Wright classification criteria for PsA that were proposed in 1973, do not offer a clear distinction between RA and PsA. The Fourni criteria that were proposed in 1999 were the first to be formulated based on patients' data; however, they require HLA-B27 positivity which excludes around $24 \%$ of patients who have the disease but test negative for HLA-B27. ClASsification for Psoriatic ARthritis (CASPAR) criteria are a congregation of international authorities who were successful in creating validated classification criteria in 2004. These criteria are highly sensitive and specific (specificity $99 \%$ and sensitivity $92 \%$ ) and have allowed for the diagnosis of PsA even in the absence of arthritis if manifestations like dactylitis and enthesitis are present. PsA can also be diagnosed despite the presence of low RF positivity. The absence of psoriasis is allowed as these criteria incorporate family history; therefore, a diagnosis can be established if other typical features are present [23].

\subsubsection{Management Guidelines}

A list of ten recommendations were proposed for the management of articular and extra-articular features of PsA. Treatments mentioned comprise of NSAIDs, synthetic DMARDs, and biological therapies. These recommendations are aimed to give a combined evidence-based and expert opinion approach to tackle this disease in the
Table 25.5 The old ACR classification criteria for systemic sclerosis [26])

\begin{tabular}{l|l}
\hline Criterion & Definition \\
\hline Major criterion & Proximal scleroderma \\
\hline \multicolumn{1}{c}{ Or two of the minor criterions: } \\
\hline Minor criteria & $\begin{array}{l}\text { 1. Sclerodactyly } \\
\text { 2. Digital pitting scars of } \\
\text { fingers or loss of the } \\
\text { distal finger pad }\end{array}$ \\
& $\begin{array}{l}\text { 3. Bilateral basilar } \\
\text { pulmonary fibrosis }\end{array}$ \\
\hline
\end{tabular}

The proposed criteria had a $97 \%$ sensitivity for definite systemic sclerosis and a $98 \%$ specificity

most optimal way and to provide the best outcomes.

Summary of European League Against Rheumatism recommendations for the management of psoriatic arthritis with pharmacological therapies (2015) [24] (Fig. 25.26).

\subsection{Systemic Sclerosis Classification Criteria and Management Guidelines}

\subsubsection{Classification Criteria of Systemic Sclerosis (Tables $25.5,25.6$ and 25.7)}

ACR developed classification criteria for systemic sclerosis (SSc) in 1980. One major and two minor criteria are required to diagnose SSc. As these criteria had a strong emphasis on skin manifestations 


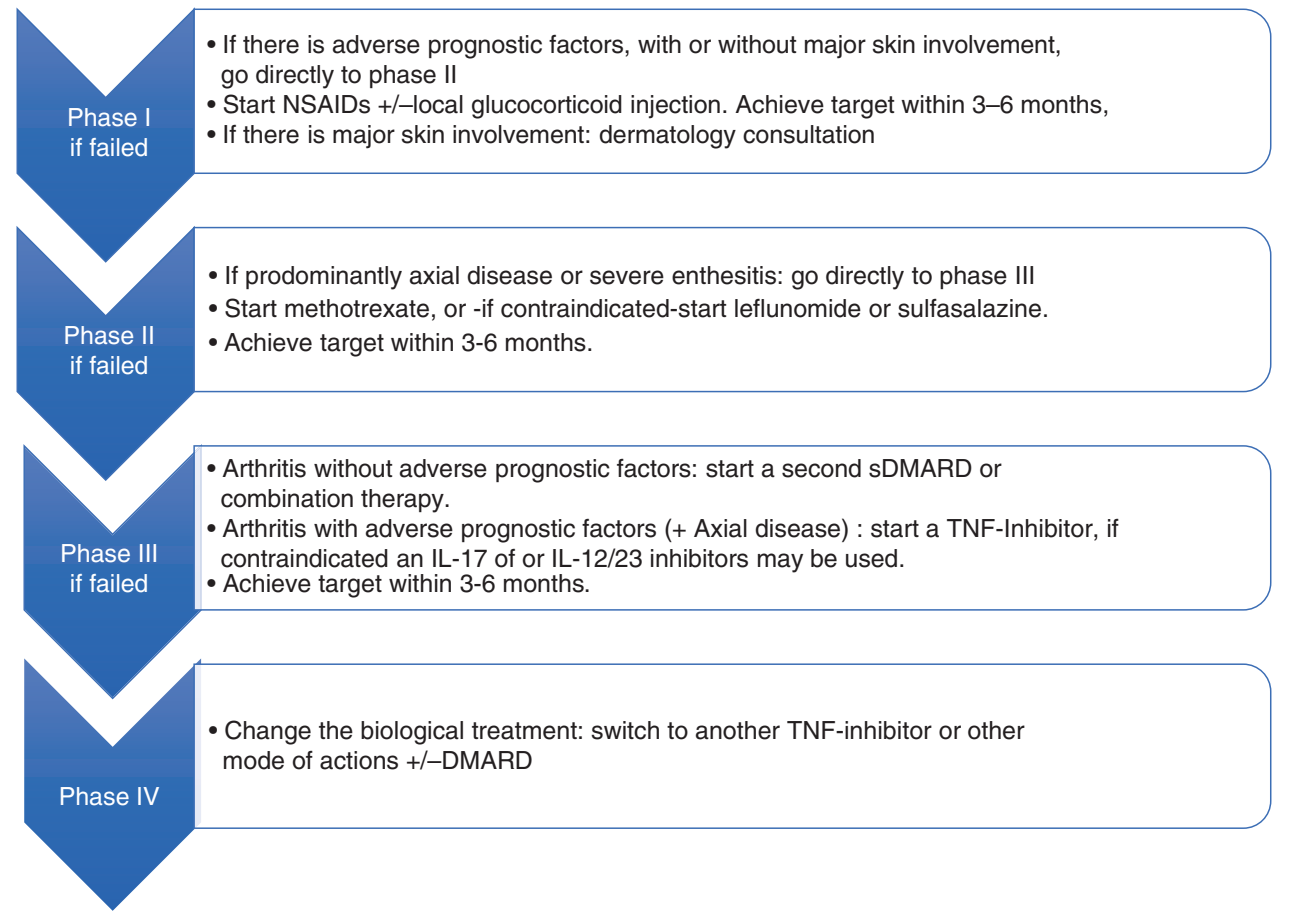

Fig. 25.26 Summary of EULAR 2015 recommendations for the management of psoriatic arthritis

Table 25.6 The American College of Rheumatology/European League Against Rheumatism criteria for the classification of systemic sclerosis $(\mathrm{SSc})^{\mathrm{a}}$

\begin{tabular}{l|l|l}
\hline Manifestation & Additional manifestation & $\begin{array}{l}\text { Weight/ } \\
\text { score }^{\mathrm{b}}\end{array}$ \\
\hline $\begin{array}{l}\text { Skin thickening of the fingers of both hands extending } \\
\text { proximal to the metacarpophalangeal joints (sufficient } \\
\text { criterion) }\end{array}$ & - & 9 \\
\hline $\begin{array}{l}\text { Skin thickening of the fingers } \\
\text { (only count the higher score) }\end{array}$ & Puffy fingers & 2 \\
\cline { 2 - 3 } & $\begin{array}{l}\text { Sclerodactyly of the fingers } \\
\text { (distal to the metacarpophalangeal joints but } \\
\text { proximal to the proximal interphalangeal } \\
\text { joints) }\end{array}$ & 4 \\
\hline Fingertip lesions (only count the higher score) & Digital tip ulcers & 2 \\
\hline & Fingertip pitting scars & 3 \\
\hline Telangiectasia & - & 2 \\
\hline Abnormal nailfold capillaries & & 2 \\
\hline $\begin{array}{l}\text { Pulmonary arterial hypertension and/or interstitial lung } \\
\text { disease (maximum score is 2) }\end{array}$ & - & 2 \\
\hline Raynaud phenomenon & - & 3 \\
\hline $\begin{array}{l}\text { SSc-related autoantibodies (anticentromere, } \\
\text { antitopoisomerase I [anti-Scl-70], anti-RNA } \\
\text { polymerase III) (maximum score is 3) }\end{array}$ & - & 3 \\
\hline
\end{tabular}

${ }^{a}$ These criteria are applicable to any patient considered for inclusion in an SSc study. The criteria are not applicable to patients with skin thickening sparing the fingers or to patients who have a scleroderma-like disorder that better explains their manifestations (e.g., nephrogenic sclerosing fibrosis, generalized morphea, eosinophilic fasciitis, scleredema diabeticorum, scleromyxedema, erthromyaglis, porphyria, lichen sclerosis, graft-versus-host disease, diabetic cheiroarthropathy)

${ }^{\mathrm{b}}$ The total score is determined by adding the maximum weight (score) in each category. Patients with a total score $\geq 9$ are classified as having definite SSc 
Table 25.7 Definitions of manifestations in the American College of Rheumatology/European League Against Rheumatism criteria of the classification of systemic sclerosis (SSc)

\begin{tabular}{l|l}
\hline Manifestation & Definition \\
\hline Skin thickening & Skin thickening or hardening not due to scarring after injury, trauma, etc. \\
\hline Puffy fingers & $\begin{array}{l}\text { Swollen digits: a diffuse, usually nonpitting increase in soft tissue mass of the digits extending } \\
\text { beyond the normal confines of the joint capsule. Normal digits are tapered distally with the } \\
\text { tissues following the contours of the digital bone and joint structures. Swelling of the digits } \\
\text { obliterates these contours. Not due to other causes such as inflammatory dactylitis. }\end{array}$ \\
\hline $\begin{array}{l}\text { Fingertip ulcers or } \\
\text { pitting scars }\end{array}$ & $\begin{array}{l}\text { Ulcers or scars distal to or at the proximal interphalangeal joint not thought to be due to } \\
\text { trauma or exogenous causes. }\end{array}$ \\
\hline \multicolumn{1}{c}{ Telangiectasiae } & $\begin{array}{l}\text { Telangiectasiae are visible macular dilated superficial blood vessels, which collapse upon } \\
\text { pressure and fill slowly when pressure is released. Telangiectasiae in a scleroderma-like } \\
\text { pattern are round and well demarcted and founds on hands, lips, inside of the mouth, and/or } \\
\text { are large mat-like telangiectasiae. Distinguishable from rapidly filling spider angiomas with } \\
\text { central arteriole and from dilated superficial vessels. }\end{array}$ \\
\hline $\begin{array}{l}\text { Abnormal nailfold } \\
\text { capillary pattern } \\
\text { consistent with } \\
\text { systemic sclerosis }\end{array}$ & $\begin{array}{l}\text { Enlarged capillaries and/or capillary loss with or without pericapillary hemorrhages at the } \\
\text { nailfold. May also be seen on the cuticle. }\end{array}$ \\
\hline $\begin{array}{l}\text { Pulmonary } \\
\text { arterial } \\
\text { hypertension }\end{array}$ & $\begin{array}{l}\text { Pulmonary arterial hypertension diagnosed by right-sided heart catheterization according to } \\
\text { standard definitions. }\end{array}$ \\
\hline $\begin{array}{l}\text { interstitial lung } \\
\text { disease }\end{array}$ & $\begin{array}{l}\text { Pulmonary fibrosis seen on high-resolution computed tomography or chest radiography, most } \\
\text { pronounced in the basilar portions of the lungs, or occurrence of "Velcro" crackles on } \\
\text { examination. }\end{array}$ \\
\hline
\end{tabular}

rather than the vascular or immunologic features that predate Raynaud's phenomenon, it became evident that it lacked proper sensitivity and specificity when it came to diagnosing early disease.

The ACR and EULAR collaborated to develop a revised classification criteria in 2013 to improve the older criteria's lower sensitivity and specificity rates in diagnosing early SSc and limited cutaneous SSc [25]. These criteria may be used for the inclusion of patients in SSc trials; however, it may be less efficient in patients with sclerodermalike syndromes.

\subsubsection{Management Guidelines of Systemic Sclerosis} (Table 25.8)

There is still a lot to be discovered in terms of the pathogenesis of SSc disorders. Treatment is challenging and no cure has yet been found. Previously, SSc trials were found to be subpar, with many based on single centers with insufficient recruitment numbers and poor randomization and control. They also did not take into account the many variable subsets and stages of the disease.
The past 10 years has witnessed significant advances in the field of SSc treatment, and many clinical trials have been documenting the efficacy of different treatment modalities. However, there are many obstacles that still stand in the way of conducting quality clinical trials, they include the following:

- SSc is an uncommon disease and can present with variable features.

- Progression rates vary between the different subsets of the disease.

- Treatment varies based on the organ that is involved.

- Disease monitoring measures are not very accurate in detecting slower incremental changes.

\subsubsection{Dermatomyositis and Polymyositis Classification Criteria and Management Guideline}

There have been many proposed classification criteria for dermatomyositis (DM) and polymyositis (PM). Brohan and Peter executed one of 
Table 25.8 Summary of 2016 EULAR recommendations for treatment of systemic sclerosis, according to the organ involvement [26]

\begin{tabular}{|c|c|c|}
\hline \multirow{3}{*}{$\begin{array}{l}\text { Digital ulcers } \\
\text { in patients with } \\
\text { SSc }\end{array}$} & $\begin{array}{l}\text { Intravenous iloprost should be considered in the treatment of digital ulcers in patients } \\
\text { with SSc. }\end{array}$ & A \\
\hline & $\begin{array}{l}\text { PDE-5 inhibitors should be considered in the treatment of digital ulcers in patients with } \\
\text { SSc. }\end{array}$ & A \\
\hline & $\begin{array}{l}\text { Bosentan should be considered for reduction of the number of new digital ulcers in SSc, } \\
\text { especially in patients with multiple digital ulcers despite use of calcium channel } \\
\text { blockers, PDE- } 5 \text { inhibitors or iloprost therapy. }\end{array}$ & A \\
\hline \multirow[t]{3}{*}{ SSc-PAH } & $\begin{array}{l}\text { Several ERA (ambrisentan, bosentan and macitentan), PDE-5 inhibitors (sildenafil, } \\
\text { tadalafil) and riociguat have been approved in the treatment of PAH associated with } \\
\text { CTDs. ERA, PDE-5 inhibitors or riociguat should be considered to treat SSc-related } \\
\text { PAH. }\end{array}$ & B \\
\hline & $\begin{array}{l}\text { Intravenous epoprostenol should be considered for the treatment of patients with severe } \\
\text { SSc-PAH (class III and IV). }\end{array}$ & A \\
\hline & $\begin{array}{l}\text { Prostacyclin analogues (iloprost, treprostinil) should be considered for the treatment of } \\
\text { patients with SSc-PAH. }\end{array}$ & B \\
\hline \multirow{3}{*}{$\begin{array}{l}\text { Skin and } \\
\text { interstitial lung } \\
\text { disease } \\
\text { (SSc-ILD) }\end{array}$} & $\begin{array}{l}\text { Methotrexate may be considered for treatment of skin manifestations of early diffuse } \\
\text { SSc. }\end{array}$ & A \\
\hline & $\begin{array}{l}\text { Despite its known toxicity, cyclophosphamide should be considered for treatment of } \\
\text { SSc-ILD, in particular for patients with SSc with progressive ILD. }\end{array}$ & A \\
\hline & $\begin{array}{l}\text { HSCT should be considered for treatment of selected patients with rapidly progressive } \\
\text { SSc at risk of organ failure. } \\
\text { In view of the high risk of treatment-related side effects and of early treatment-related } \\
\text { mortality, careful selection of both patients and experienced medical teams are of key } \\
\text { importance. }\end{array}$ & A \\
\hline \multirow{2}{*}{$\begin{array}{l}\text { Scleroderma } \\
\text { renal crisi } \\
(\mathrm{SRC})\end{array}$} & Experts recommend immediate use of ACE inhibitors in the treatment of SRC. & $\mathrm{C}$ \\
\hline & $\begin{array}{l}\text { Because several retrospective studies suggest that glucocorticoids are associated with a } \\
\text { higher risk of SRC, blood pressure and renal function should be carefully monitored in } \\
\text { patients with SSc treated with glucocorticoids. }\end{array}$ & $\mathrm{C}$ \\
\hline \multirow{3}{*}{$\begin{array}{l}\text { SSc-related } \\
\text { gastrointestinal } \\
\text { disease }\end{array}$} & $\begin{array}{l}\text { Experts recommend that PPI should be used for the treatment of SSc-related GERD and } \\
\text { for the prevention of oesophageal ulcers and strictures }\end{array}$ & $\mathrm{C}$ \\
\hline & $\begin{array}{l}\text { Experts recommend that prokinetic drugs should be used for the management of } \\
\text { SSc-related symptomatic motility disturbances (dysphagia, GERD, early satiety, } \\
\text { bloating, pseudo-obstruction, etc). }\end{array}$ & $\mathrm{C}$ \\
\hline & $\begin{array}{l}\text { Experts recommend the use of intermittent or rotating antibiotics to treat symptomatic } \\
\text { small intestine bacterial overgrowth in patients with SSc. }\end{array}$ & $\mathrm{D}$ \\
\hline
\end{tabular}

CTD, connective tissue disease; ERA, endothelin receptor antagonists; EULAR, European League against Rheumatism; GERD, gastro-oesophageal reflux disease; HSCT, haematopoietic stem cell transplantation; PAH, pulmonary arterial hypertension; PDE-5, phosphodiesterase type 5; PPI, proton pump inhibitor; SRC, scleroderma renal crisis; SSc, systemic sclerosis; SSc-RP, Raynaud's phenomenon in patients with SSc

the earliest criteria in 1975 , which had been used to aid in research for several decades. These criteria demanded the presence of typical skin manifestations and at least three out of four other criteria to meet the diagnosis of DM. Patients who were diagnosed with PM had to have met all four criteria in addition to the cutaneous features. As there are no highly specific autoantibodies, biopsy and histological proof remain important diagnostic tools. There are limitations with these criteria as research studies conducted on PM/DM are scarce, making the classification of these diseases difficult in addition to the poor understanding of the relationship between DM and PM.

The discovery of at least eight antisynthetase autoantibodies had allowed for significant advances in diagnosing DM and PM, especially as these autoantibodies were all associated with different manifestations of the disease (see chapter "Diagnostic Approach to Proximal Myopathy"). 


\subsubsection{Summary of Polymyositis and Dermatomyositis Classification Criteria [27]}

(Fig. 25.27)

Management of inflammatory myositis is difficult due to the scarcity of randomized controlled trials and the fact that the disease is very uncommon. Treatment includes:

- Glucocorticoids, immunoglobulins, and the newly included mycophenolate mofetil.

- Mycophenolate mofetil and rituximab which were found to be efficient in refractory cases.

- Anti-tumor necrosis factor (anti-TNF) inhibitors which were also found to be useful in treating resistant cases.

In general, there are limited data upon which the base treatment recommendations for DM and PM are provided.

\subsubsection{Sjögren's Syndrome Classification Criteria and Management Guidelines} (Table 25.9)

The ACR proposed classification criteria for Sjögren's syndrome (SS). These criteria were based on evidence from the Sjogren's International Collaborative Clinical Alliance (SICCA) and also on the knowledge of field authorities; it is easily applicable and based mostly on objective testing.

At least two of the following items need to be present to diagnose SS:

- Positive serum anti-SSA and/or anti-SSB or positive rheumatoid factor plus antinuclear antibodies $\geq 1: 320$.

- Ocular staining score $\geq 3$.

- Labial salivary gland biopsy showing focal lymphocytic sialadenitis with a focus score $\geq$ 1 foci/4 $\mathrm{mm}^{2}$ [28].

Table 25.9 ACR classification criteria for Sjogren's syndrome

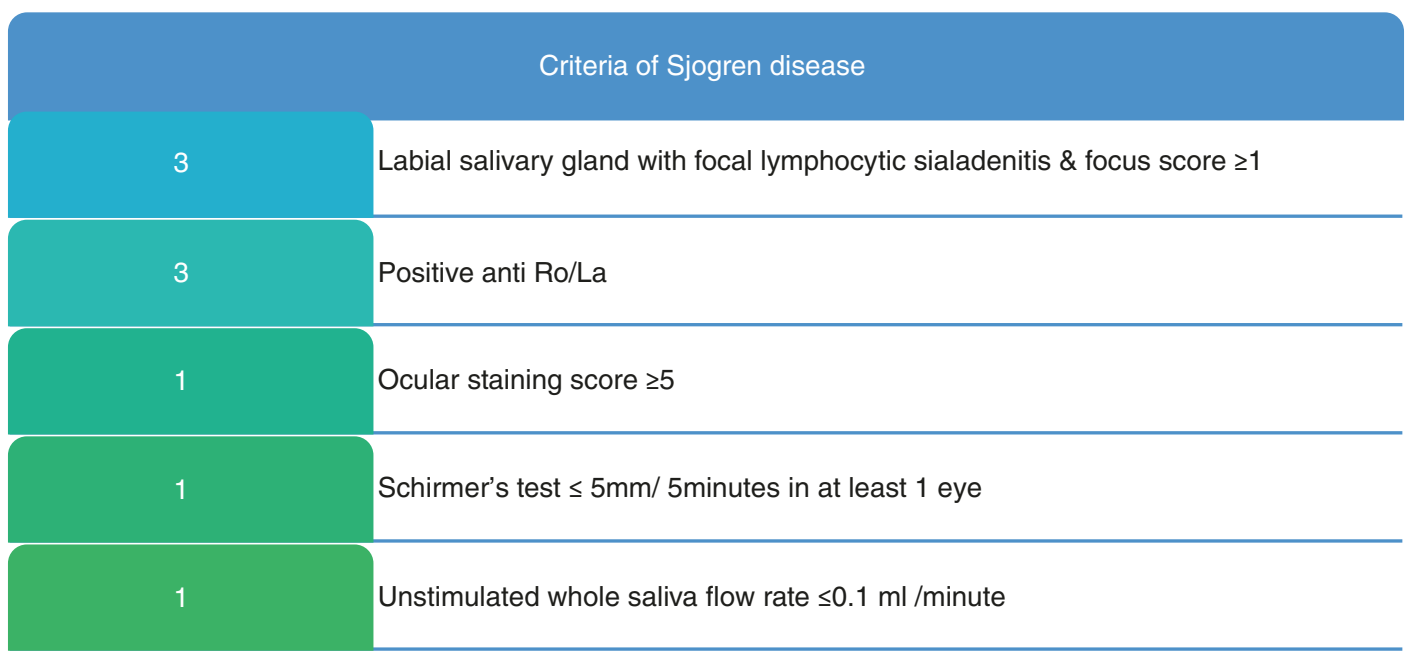

- The criteria applies to those who meet the inclusion criteria with a score of at least 5 and to those who do not fulfil any of the exclusion criteria

- Exclusion criteria include:

- History of head and neck radiation treatment

- Active hepatitis c infection

- AIDS

- Sarcoidosis

- Amyloidosis

- Graft-verus-host diease

- IgG4-related diease 


\section{Criteria for Diagnosis of Polymyositis and Dermatomyositis}

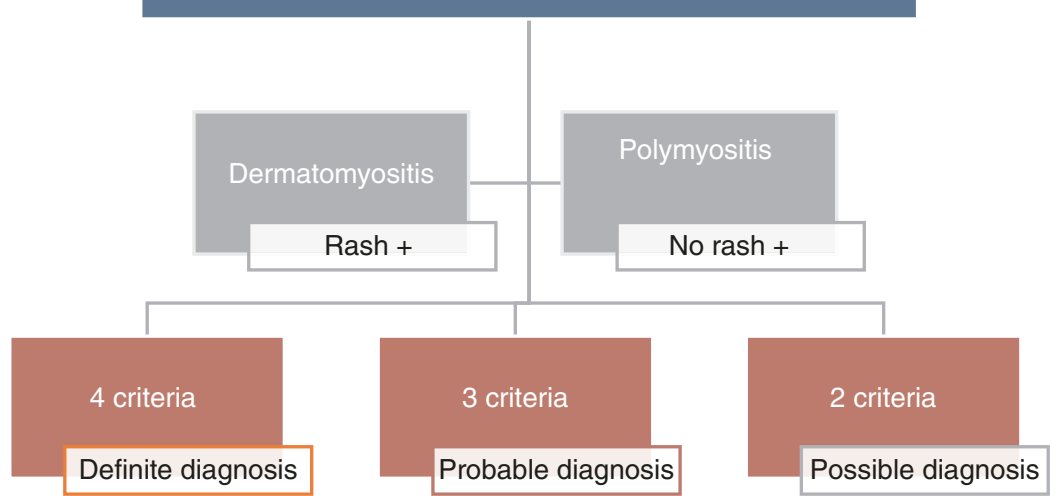

\begin{tabular}{ll}
\hline \multirow{2}{*}{$\begin{array}{l}\text { Inclusion } \\
\text { criteria: }\end{array}$} & \multicolumn{1}{l}{ Symmetrical proximal muscle weakness } \\
\cline { 2 - 2 } & Elevated muscle enzymes \\
\hline Characteristic EMG abnormalities & Necrosis, phagocytosis, regeneration and inflammtion in the muscle biopsy \\
\hline Gottron's sign or heliotrope rash or papules in dermatomyositis \\
criteria:
\end{tabular}

Fig. 25.27 Criteria for the diagnosis of polymyositis and dermatomyositis

There is no single medication available that has been proven to be effective in SS in randomized controlled trials. Treatment at this moment is mainly symptomatic, supportive, and empiric.

In a subset of patients in which arthralgia is a major symptom, some clinicians prescribe antimalarials (hydroxychloroquine). More recently, biological therapies are studied for their potential efficacy in early SS. Therefore, proper patient detection in the early stages of the disease is important.

B-cell targeting with either rituximab or belimumab has been studied, and a study with epratuzumab is planned for the near future [29]. Until now, no immunomodulatory drugs have been proved to be effective in primary SS.

\subsubsection{Behcet's Disease Classification Criteria and Management Guidelines}

Behcet's disease (BD) is an inflammatory disease that runs a relapsing and remitting course. The pathogenesis is not entirely known and no conclusive diagnostic tests have been found. The diagnosis relies on clinical grounds.

A group of physicians responsible for the treatment of large numbers of patients with BD formed the International Study Group (ISG) and published the ISG criteria for diagnosis in 1990. The utility of the criteria is dependent on the prevalence of the syndrome in the background population; there may also be atypical patients 
who do not fulfill the criteria. These criteria are meant for the classification of groups of patients participating in research programs to ensure comparability of the groups, and not for the diagnosis of the individual patients in clinical situations.

\subsubsection{Diagnostic Criteria for Behcet's Disease, International Study Group for Behcet's Disease (1990) (Tables 25.10 and 25.11)}

Treatment of BD is mainly based on evidence that were gleaned from case reports and case series with a paucity of randomized controlled trials. Management relies both on organ dysfunction and on the degree of dysfunction. As patients mostly have multisystemic dysfunction, management is thus dictated by the most critical organ that is involved.

Since 1998, there have been only a small number of advances in the quality of BD literature. These include significant benefits found in randomized trials of colchicine, mucocutaneous disease, and the introduction of anti-TNF-alpha therapy. Most current approaches with other medications are dictated primarily by extrapola- tion of the use of certain medications from their efficacy in other inflammatory conditions.

The EULAR and the task force of the EULAR Standing Committee for Clinical Affairs (ESCCA) proposed a list of treatment recommendations in 2008. This list was primarily derived from a systematic review that was conducted in 2006. The treatments of all facets of BD were outlined in nine recommendations. Recommendations regarding the management of ocular, mucocutaneous, and musculoskeletal were based on stringent evidence. However, manifestations that affect the vascular, neurological, and gastrointestinal systems were derived from data that is of a lesser quality, including open trials, observational studies, and expert opinions. There is a significant need for more research to cover all areas that are deficient in this disease [30].

\subsubsection{Gout Classification Criteria and Management Guidelines [31] (Box 25.2)}

Gout is considered to be one of the most common inflammatory arthritis. It is characterized by the deposition of monosodium urate crystals in the extracellular fluid. The diagnosis of gout is sug-

Table 25.10 Diagnosis criteria for Behcet's disease

\begin{tabular}{|l|l|}
\multicolumn{1}{c}{ Criteria of Behcet's diease } \\
\hline 2 & Ocular lesions \\
\hline 2 & Senital aphthosis \\
\hline 1 & Skin lesion \\
\hline 1 & Neurological manifestations \\
\hline 1 & Vascular manifestations \\
\hline
\end{tabular}

- *4 points or more diagnose Behcet diease 
Table 25.11 EULAR recommendations for the management of Behcet's disease

\begin{tabular}{|c|c|}
\hline \multicolumn{2}{|r|}{ Managment of Behcet's diease } \\
\hline $\begin{array}{c}\text { Arthritis /ertyhaema } \\
\text { nodosum }\end{array}$ & Cholchicine \\
\hline Posterior eye diease & Corticosteriod \& azathioprine(AZA) \\
\hline Retinal diease & Ciclosporine or infliximab \pm corticosteriod \& AZA \\
\hline Venous thrombosis & Immunosuppressive \\
\hline $\begin{array}{c}\text { Pulmonary or arterial } \\
\text { aneurysms }\end{array}$ & Cyclophosphamide and corticosteriod \\
\hline GI involvment & Sulfasalazine, corticosteriod, azathioprine, TNF $\alpha$ antagonist before surgery \\
\hline CNS inlvoment & $\begin{array}{l}\text { Corticosteriod, AZA, TNF } \alpha \text { antagonist cyclophosphamide } \\
\text { Ciclosporin A not recomended }\end{array}$ \\
\hline Resistant cases & AZA, IFN $\alpha$ and TNF $\alpha$ antagonist \\
\hline
\end{tabular}

- *2008 EULAR recommendations of management for Behcet disease

gested by the presence of typical clinical features along with increased urate levels in the serum. However, coincidentally found high serum urate concentrations could also occur in other causes of acute arthritis.

The 2014 guidelines [31] provide practical recommendations which are supported by evidence-based practice in addition to the opinions of a large number of multinational expert rheumatologists. This is called the $3 e$ (Evidence, Expertise, Exchange) Initiative.

In these recommendations, they emphasize the finding of monosodium urate crystals in synovial fluid as a crucial step for definitive diagnosis of gout.

The 3e Initiative differs from the 2012 ACR guidelines in two recommendations:

1. Kidney function should be assessed in patients with high uric acid levels and/or gout. Measurements of the patient's cardiovascular risk factors are also suggested. In the previous 2012 guideline, it was not necessary to assess for cardiovascular risk factors or renal function status.

2. It was suggested that allopurinol be used as the first-line urate-lowering therapy.

All xanthine oxidase inhibitors were previously considered as first-line choices for therapy. However, now allopurinol is the first-line agent with alternatives including uricosurics or febuxostat. The use of uricase on its own can be used in severe and refractory cases where other lines were either exhausted or contraindicated.

It is important to mention the limitations of these guidelines. Three limitations were mentioned: first, there were no participants from other specialties like nephrology so, the applicability of these recommendations is not clear. Second, many recommendations have different statements with variable degrees of supporting evidence. Lastly, agreement on these recommendations was variable which suggests some degree of dispersion. However, around more than $80 \%$ of rheumatologists voted in support of these recommendations. 


\section{Diagnosis management of Gout artheritis}

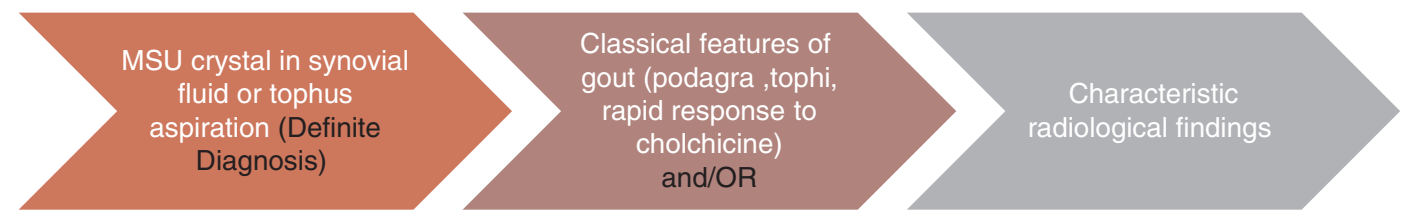

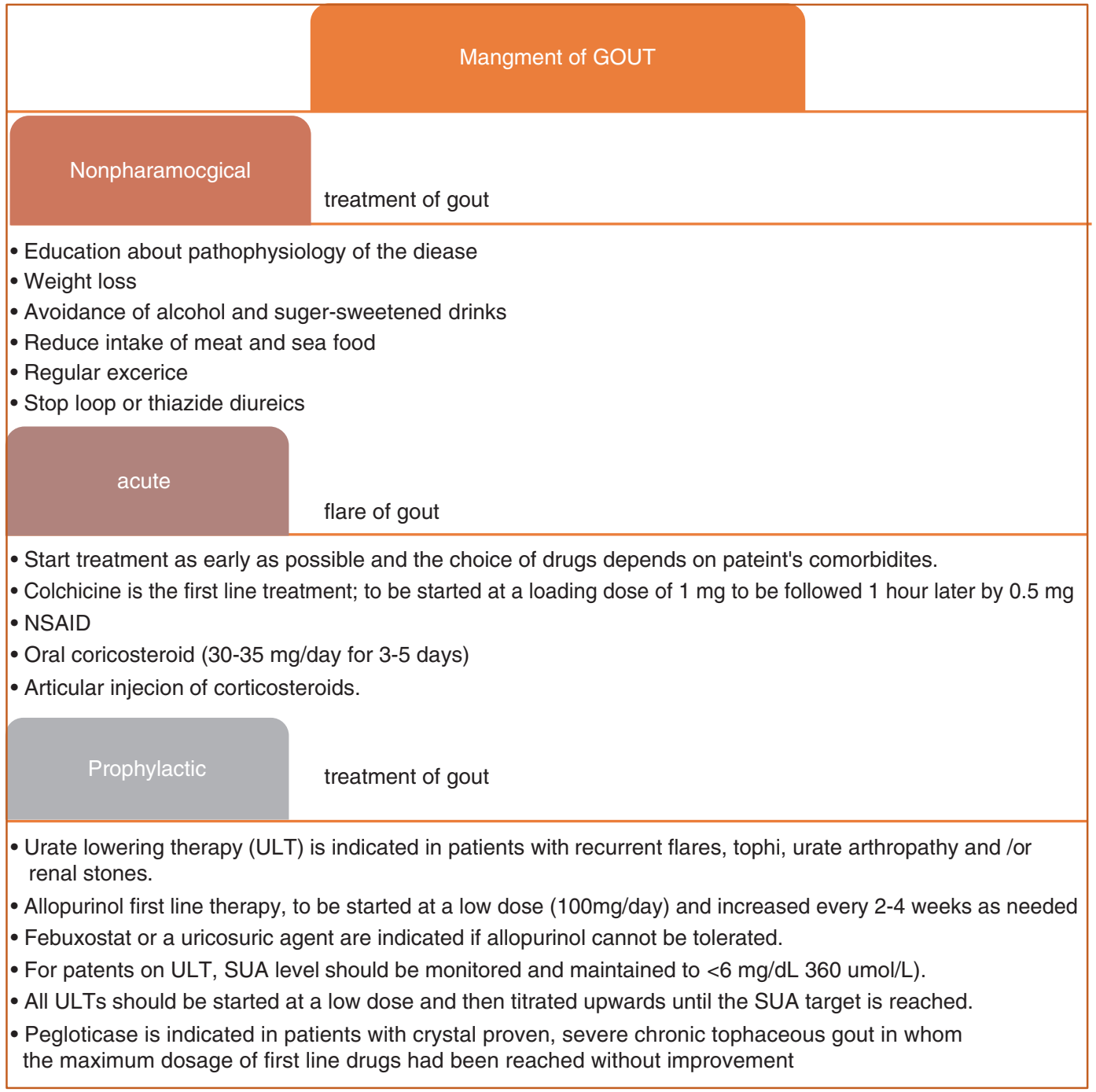

Box 25.2: 2016 EULAR recommendation of gout management

2016 EULAR recommendation of gout management

Box 25.2 Multinational Recommendations on the Diagnosis and Management of Gout 


\subsection{Osteoarthritis Classification Criteria and Management Guidelines}

\subsubsection{Classification Criteria} (Fig. 25.28)

Osteoarthritis (OA) is a chronic musculoskeletal disease that often leaves the patients suffering from pain and disability. OA may be classified as primary or secondary. Optimal management requires early diagnosis.

The ACR formulated classification criteria for $\mathrm{OA}$, and although they are highly specific when applied and allow for the discernment between patients with inflammatory arthritis and patients with osteoarthritis, they have lower sensitivity rates, especially if the differentiation between

Knee osteoarthritis $(\mathrm{OA})$

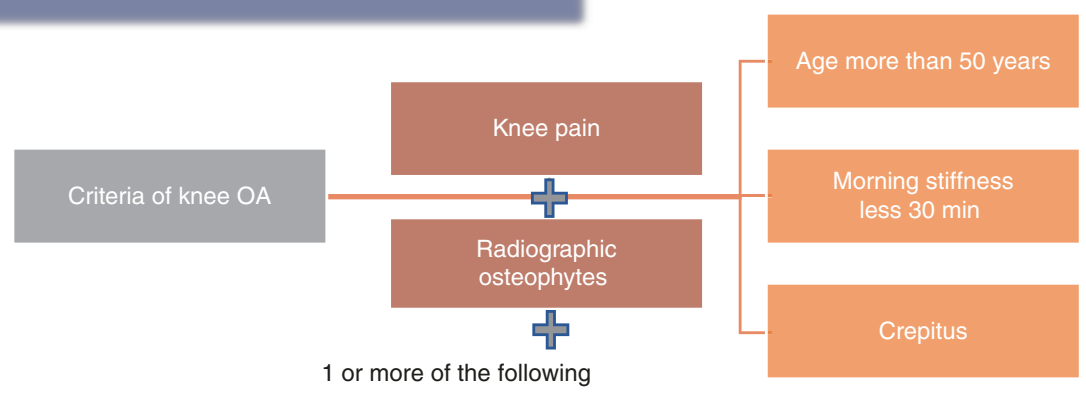

Hand osteoarthritis (OA)

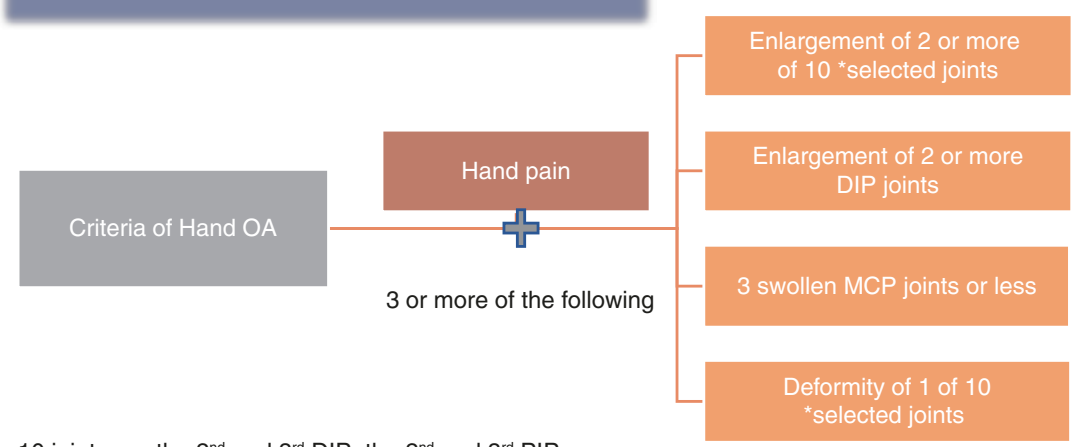

*The 10 joints are the $2^{\text {nd }}$ and $3^{\text {rd }}$ DIP, the $2^{\text {nd }}$ and $3^{\text {rd }}$ PIP and the first $\mathrm{CMC}$ joints

Hip osteoarthritis (OA)

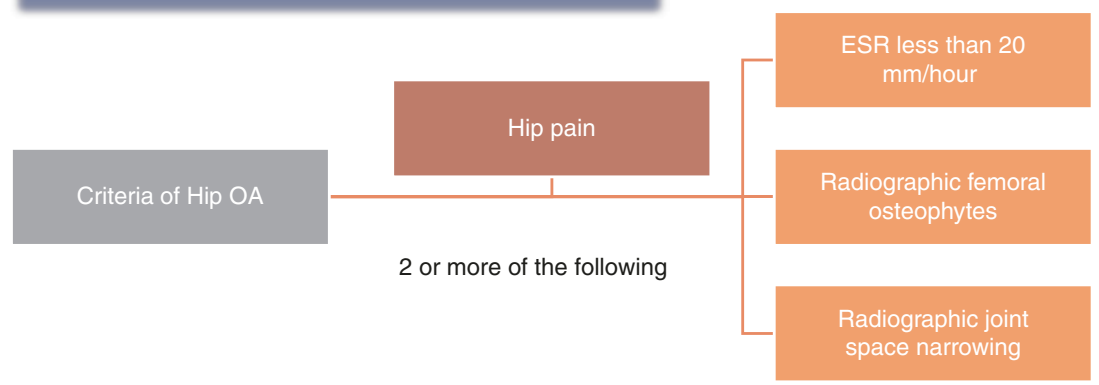

Fig. 25.28 ACR classification criteria for osteoarthritis (OA) [32, 33, 34] 
patients who are in the early stages of their disease and healthy subjects is sought.

Another set of recommendations was developed by the EULAR which showed that hand and knee OA can be diagnosed using clinical assessment alone. The EULAR guidelines include ten recommendations which were derived from a systematic review of literature and the opinions of experts in the field. They stipulate that the presence of symptoms including knee pain, brief morning stiffness, and disturbances in functionality along with clinical signs including knee crepitus, limitation of range of motion, and enlargement of bones would suffice to make a diagnosis without the need to proceed for any imaging modalities. This approach would benefit primary care physicians the most. However, plain radiographs and further imaging can be done if the presentation is atypical or other differentials are considered.

\subsubsection{Osteoarthritis Management Guidelines [35] (Figs. 25.29, 25.30 and 25.31)}

Research regarding the management of osteoarthritis is still scarce. The disease is primarily managed using a consensus of opinions from experts in the field. The EULAR has formulated a list of treatment recommendations in 2010 which was derived from both experts' opinions and research evidence. The list covers the treatment of the hand, hip, and knee OA. In determining the strength of recommendation for any treatment, many factors other than efficacy need to be considered, including safety, cost, logistics of delivery, and the individual patient's acceptability.

\subsubsection{Osteoporosis Classification Criteria and Management Guidelines [36-41]}

(Table 25.12) (Box 25.3)

(Fig. 25.32)

Osteoporosis is characterized by decreased bone mass and disruption in musculoskeletal microarchitecture leading to bone fragility and higher risks of fracture. The disease often remains undetected until a fracture develops. Osteoporosis is confirmed either by the occurrence of a fragility fracture in the hip or spine or by confirmation of decreased bone density by bone mineral density (BMD) measurements. The definitions of osteopenia and osteoporosis based on BMD testing were defined by the World Health Organization (WHO).

Risk stratification for osteoporosis is imperative in all adults. BMD-independent factors that should be kept in consideration include older age, previous occurrences of fragility fractures, use of steroids, smoking and alcohol consumption, and a positive family history of fracture. There are recommendations about when to do BMD screening to detect osteoporosis and when to repeat BMD testing.

The Fracture Risk Assessment Tool (FRAX) was proposed by the WHO in 2008; this tool helps to determine the 10-year risk of hip fractures or major fragility fractures. However, as the association between decreased bone mass and fractures in premenopausal women is not as well studied as the one in postmenopausal women, bone mineral density criteria and management guidelines may not be as useful in the premenopausal women population.

Ruling out secondary causes of osteoporosis is imperative. Management guidelines include non-pharmacological lines of therapy and phar- 
Non pharmacologic recommendations for the management of Knee OA

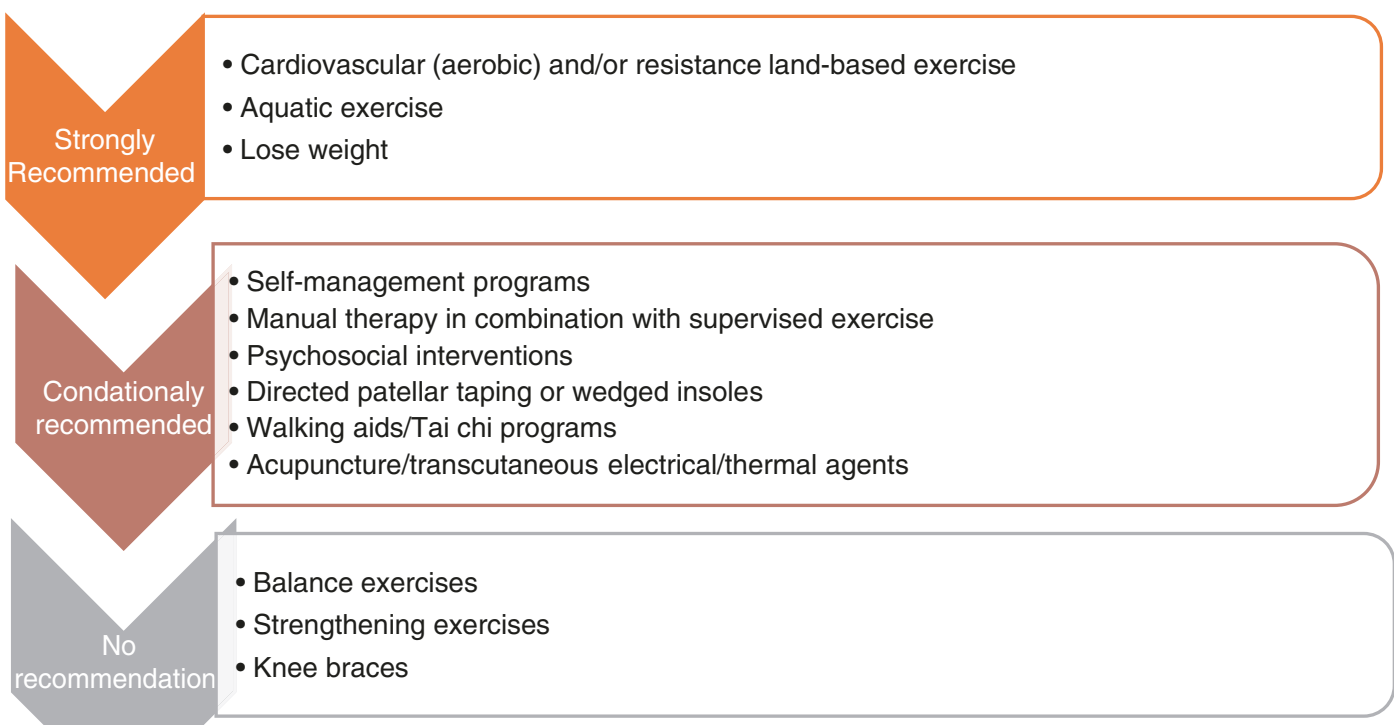

Pharmacologic recommendations for the management of Knee OA

\begin{tabular}{|c|c|}
\hline Recommended & $\begin{array}{l}\text { - Acetaminophen } \\
\text { - Oral/Topical NSAIDs } \\
\text { - Tramadol } \\
\text { - Intraarticular corticosteriod injections }\end{array}$ \\
\hline $\begin{array}{l}\text { Conditionalyy } \\
\text { recommended }\end{array}$ & $\begin{array}{l}\text { - Chondroitin sulfate } \\
\text { - Glucosamine } \\
\text { - Topical capsaicin }\end{array}$ \\
\hline $\begin{array}{l}\text { No } \\
\text { recommendation }\end{array}$ & $\begin{array}{l}\text { - Intraarticular hyaluronic acid } \\
\text { - Duloxetine } \\
\text { - Opioid }\end{array}$ \\
\hline
\end{tabular}

Fig. 25.29 Nonpharmacologic and pharmacological recommendations for the management of knee $\mathrm{OA}^{*}$ 
Nonpharmacologic recommendations for the management of Hand OA

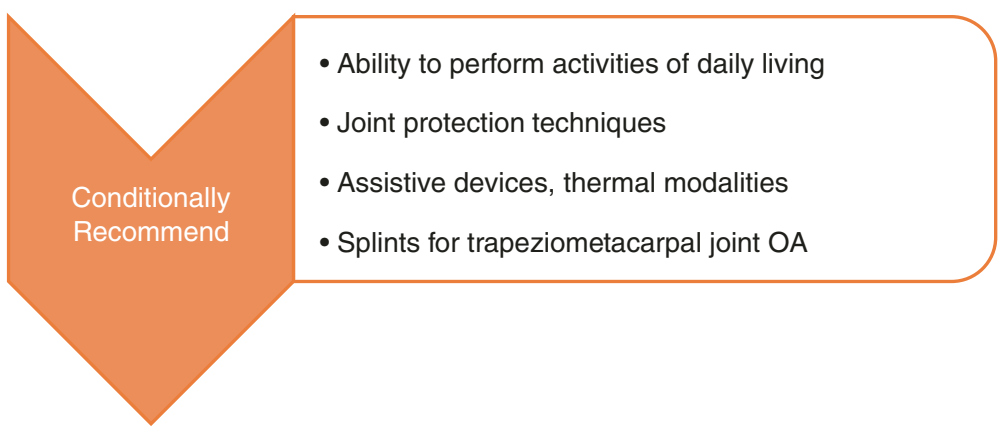

Pharmacologic recommendations for the management of Hand OA

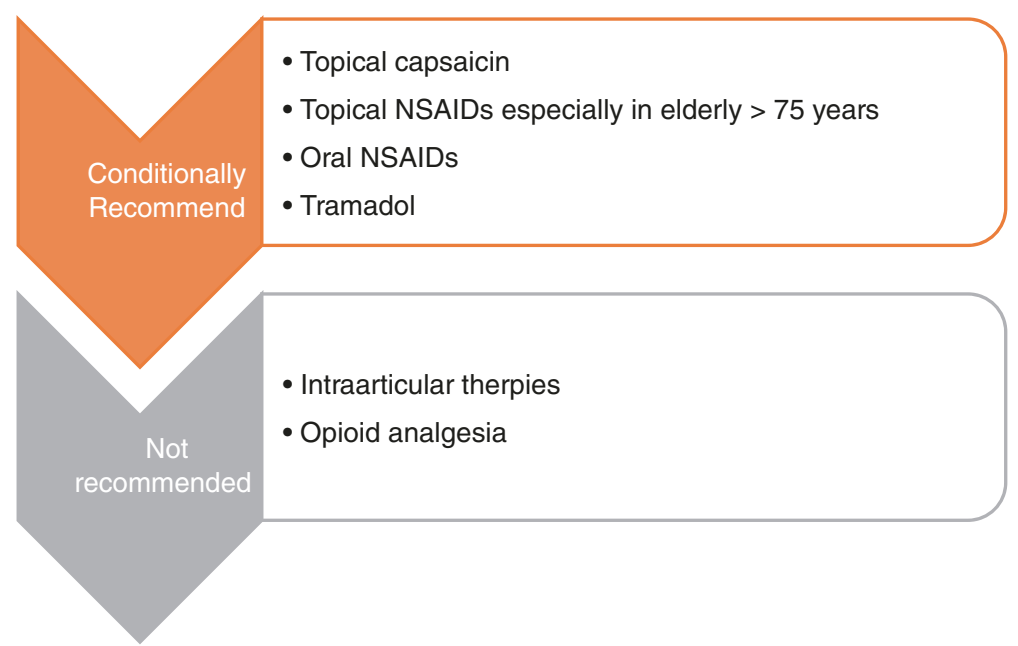

Fig. 25.30 Nonpharmacologic and pharmacological recommendations for the management of hand OA 
Nonpharmacologic recommendations for the management of Hip OA

- Cardiovascular (aerobic) and/or resistance land-based exercise

- Aquatic exercise

Strongly

Recommended

- Loss weight

- Self-management programs

- Manual therapy in combination with supervised exercise

- Psychosocial interventions

Condationaly

- Walking aids as needed

- Thermal agents

- Balance exercises

- Strengthening exercises

- Manual therpy alone

- Tai chi

Pharmacologic recommendations for the management of Hip OA

- Acetaminophen

- Oral NSAIDs

- Tramadol

Conditionally

recommend

- Intraarticular corticosteriod injections

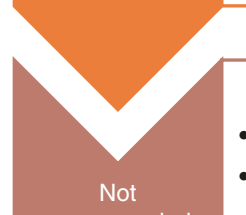

- Chondroitin sulfate

recommended

- Glucosamine

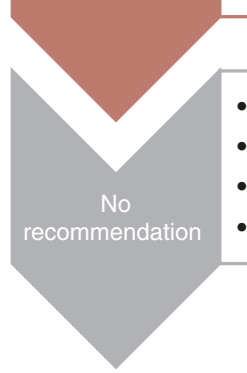

- Intraarticular hyaluronate injection

- Topical NSAIDs

- Duloxetine

- Opioid analgesia

Fig. 25.31 Nonpharmacologic and pharmacological recommendations for the management of hip OA* 
Table 25.12 Defining osteoporosis by BMD WHO definition of osteoporosis based on BMD

\begin{tabular}{l|l|l|l|l}
\hline Classification & Normal & Osteopenia & Osteoporosis & Severe Osteoporosis \\
\hline $\begin{array}{l}\text { BMD ( the SD of a young adult } \\
\text { reference population ) }\end{array}$ & $\begin{array}{l}\text { Within } 1 \\
\mathrm{SD}\end{array}$ & $\begin{array}{l}1 \text { to } 2.5 \text { below } \\
\text { the SD }\end{array}$ & $\begin{array}{l}2.5 \text { or more below } \\
\text { the SD }\end{array}$ & $\begin{array}{l}2.5 \text { or more below the } \\
\text { SD }\end{array}$ \\
\hline $\begin{array}{l}\text { T-score } \\
\text { above }\end{array}$ & -1 to -2.5 & -2.5 and below & $\begin{array}{l}-2.5 \text { and below with a } \\
\text { fracture }\end{array}$ \\
\hline
\end{tabular}

Box 25.3 Factors That Identify People Who Should Be Assessed for Osteoporosis

Fig. 25.32 Indications for BMD testing

\section{Risk Factors of Osteoporosis}

- Age above 65 years

- Low body weight

- Early menopause

- Fractures: vertebral compression fracture, fragility fracture, family history of osteoporotic fracture.

- Disease: malabsorption syndromes, primary hyperparathyrodism, hypogonadism, rheumatoid arthritis, clinical hyperthyroidism.

- Medications: systemic glucocorticoid disease for more than 3 months, prolonged anticonvulsant therapy, chronic heparin therapy.

- Dietary: low calcium intake, excessive alcohol intake, excessive caffeine intake.

- Smoker

- Propensity to fall

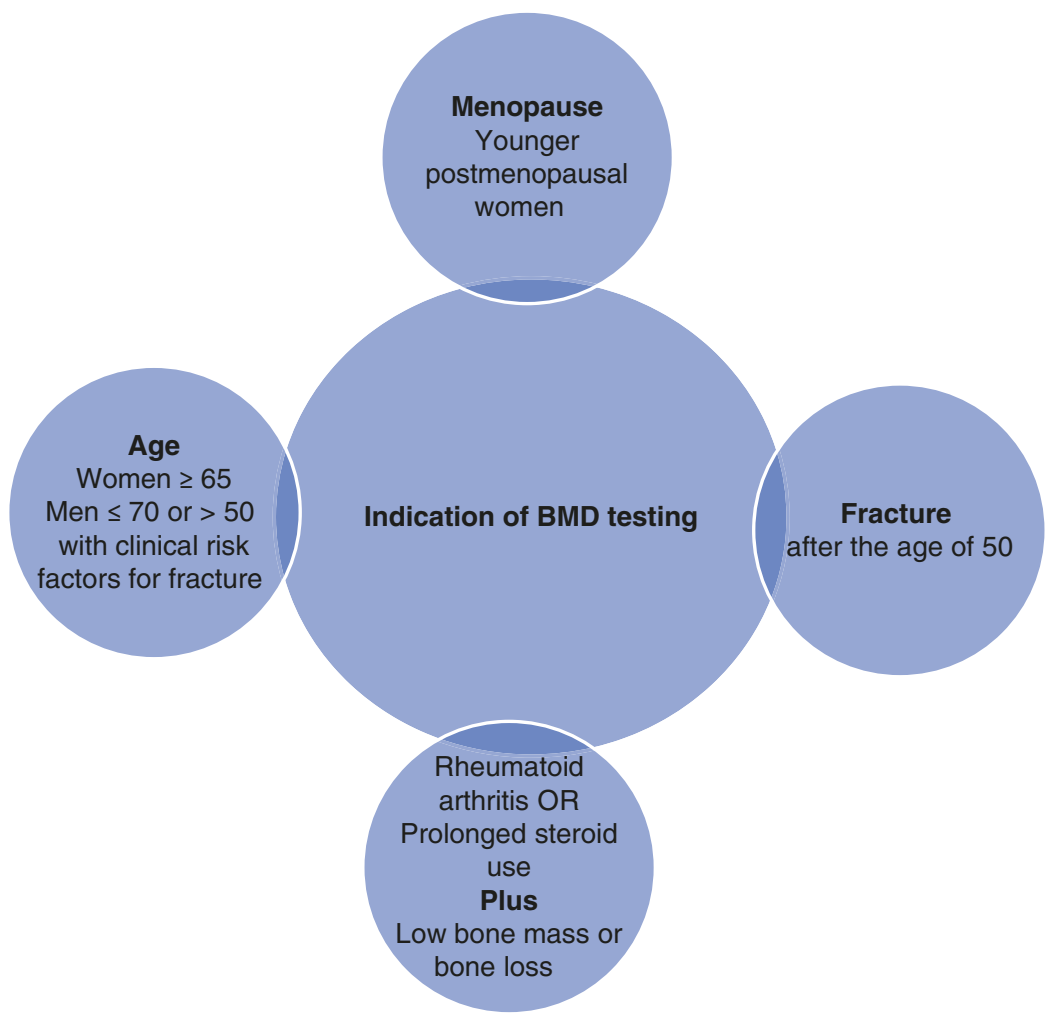


Fig. 25.33 Consider medical therapies based on the following

Fig. 25.34 Consider nonmedical therapeutic interventions
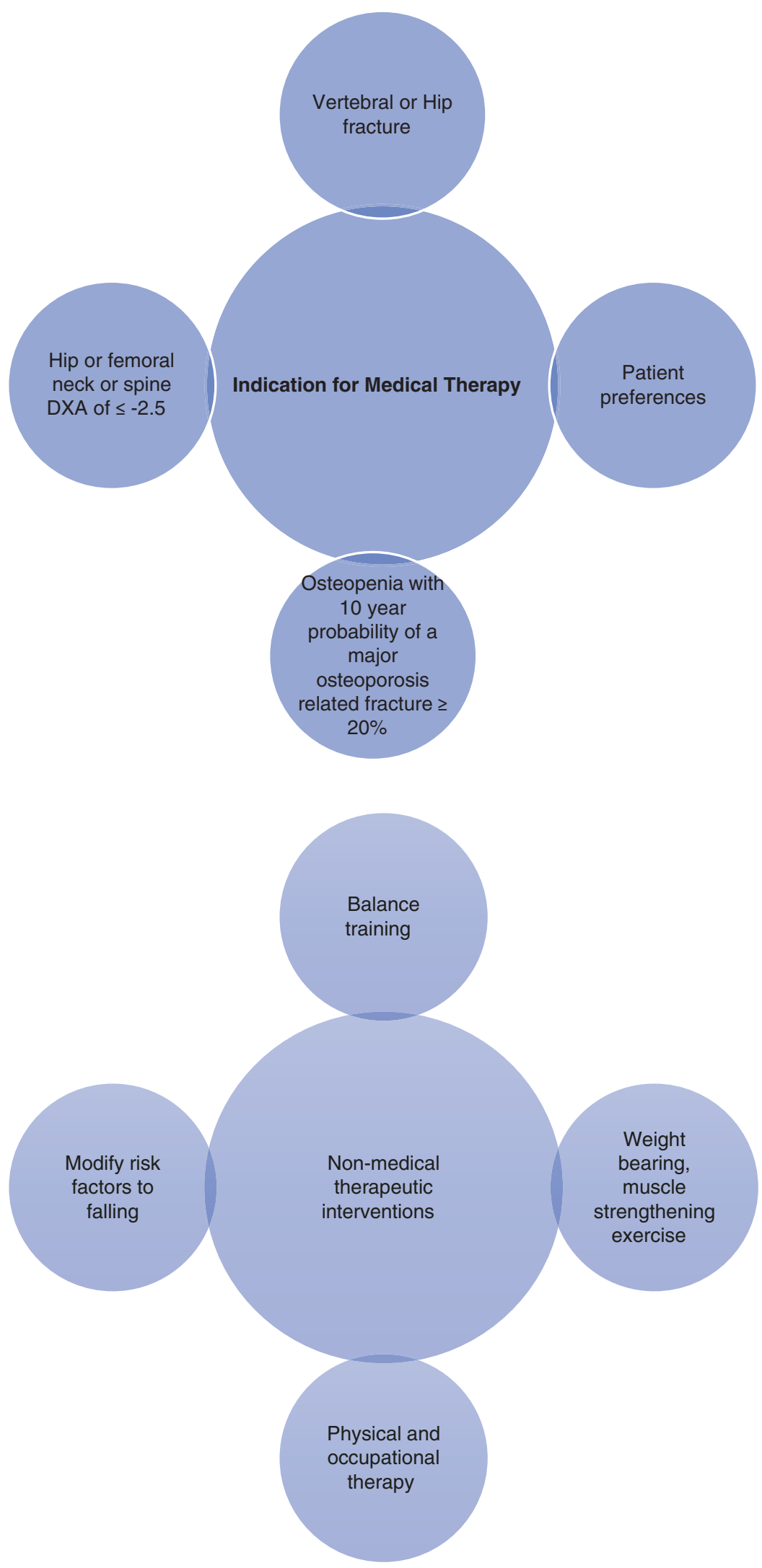
Fig. 25.35 Follow-up recommendations

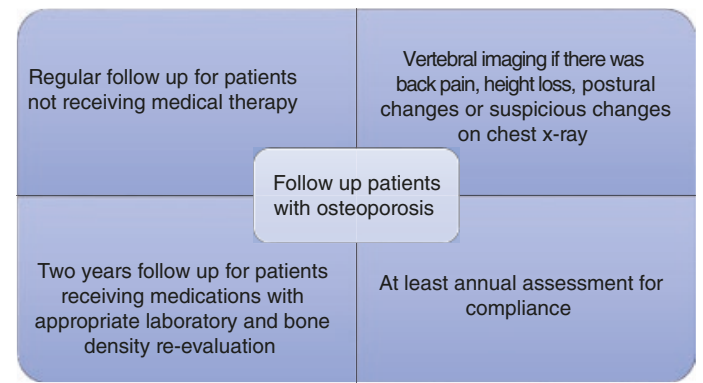

Approach to management of Osteoporosis
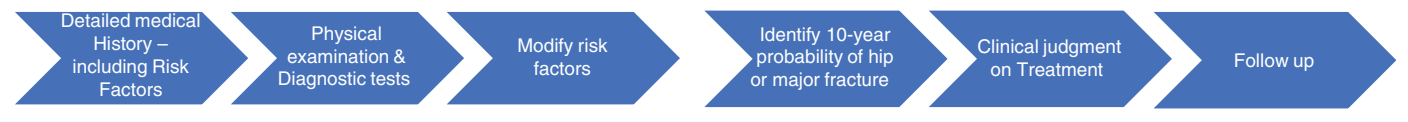

Fig. 25.36 Clinical approach to managing osteoporosis in postmenopausal women and men age 50 and older general principles (2013)

\section{Osteoporosis Treatment Strategies}

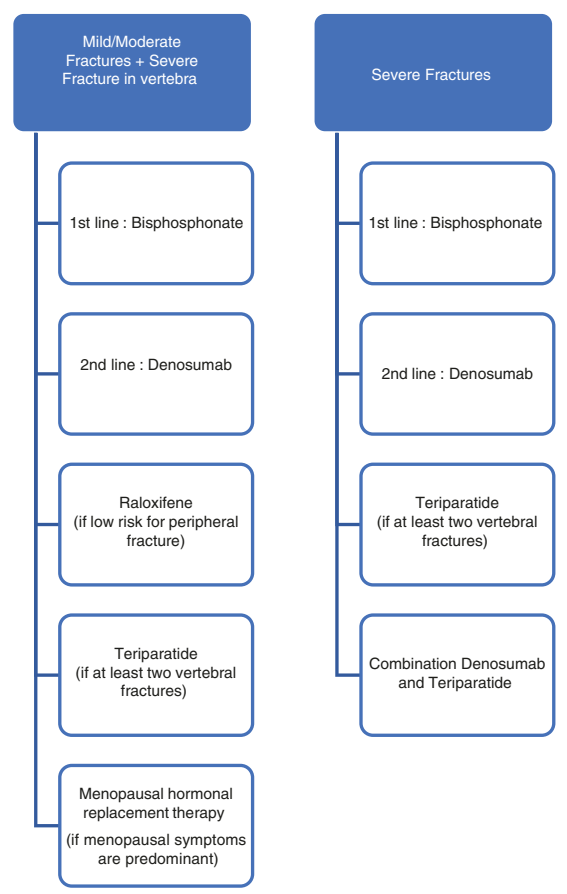

Fig. 25.37 Pharmacological treatment of postmenopausal osteoporosis macological agents. Assessment and treatment of preventable risk factors is also advised.

Universal recommendations for all patients (Figs. 25.33, 25.34, 25.35, 25.36 and 25.37) (Box 25.4) include:

- Proper calcium and vitamin D dietary intake.

- Management of vitamin D deficiency.

- Weight-bearing exercises and exercises to improve muscle strength.

- Prevention of fall.

- Smoking cessation and limitation of alcohol consumption.

The treatment guidelines mentioned should be thought of as a guide in clinical practice. A thorough consideration of each patient's situation is imperative in making proper management decisions. These treatment guidelines should not stop physicians from offering therapies to those who do not meet the BMD (T-score $\leq-2.5$ ) and FRAX diagnostic scores, or are not at a high enough risk of fracture despite decreased BMD, as every patient's needs should be assessed individually. 
Box 25.4 Treatment

Strategies for

Postmenopausal

Osteoporosis

\author{
Indications \\ for \\ treatment of \\ osteoporosis \\ related \\ fractures
}

Severe fractures ( vertebral fracture, proximal or distal femur, proximal humerus, pelvic and others ) with $\mathrm{T}$-score $\leq-1$

Non-severe fractures with T-score $\leq-2$

Risk factors for osteoporosis or high fall risk with T-score $\leq-3$

Risk factors for osteoporosis or high fall risk, T-score $\geq-3$ and medical therapy is indicated by FRAX score.

Acknowledgments The authors would like to thank Narges Omran, Abdullah Almajnoni, Samar Barnawi, and Lina Alkibbi for their contributions to this chapter in the previous edition.

\section{References}

1. Britsemmer K, Ursum J, Gerritsen M, et al. Validation of the 2010 ACR/EULAR classification criteria for rheumatoid arthritis: slight improvement over the 1987 ACR criteria. Ann Rheum Dis. 2011;70:1468-70.

2. Smolen JS, Landewé R, Bijlsma J, et al. EULAR recommendations for the management of rheumatoid arthritis with synthetic and biological diseasemodifying antirheumatic drugs: 2016 update. Ann Rheum Dis. 2017;76:960-77.

3. Hochberg MC. Updating the American College of Rheumatology revised criteria for the classification of systemic lupus erythematosus. Arthritis \& Rheumatism. 1997;40(9):1725.

4. Petri, Michelle, et al. "Derivation and validation of systemic lupus international collaborating clinics classification criteria for systemic lupus erythematosus." Arthritis and Rheumatism. Vol. 63. No. 10. Wiley-Blackwell, 2011

5. Fanouriakis A, et al. 2019 update of the EULAR recommendations for the management of systemic lupus erythematosus. Ann Rheum Dis. 2019; annrheumdis-2019

6. Hahn BH, et al. American College of Rheumatology guidelines for screening, treatment, and management of lupus nephritis. Arthritis care \& research. 2012;64.6:797-808.

7. Weening JJ, et al. The classification of glomerulonephritis in systemic lupus erythematosus revisited. Journal of the American Society of Nephrology. 2004;15.2:241-50.

8. Furness PN, Taub N. Interobserver reproducibility and application of the ISN/RPS classification of lupus nephritis - a UK-wide study. Am J Surg Pathol. 2006;30(8):1030-5.
9. Liang $\mathrm{MH}$, et al. The American College of Rheumatology response criteria for proliferative and membranous renal disease in systemic lupus erythematosus clinical trials. Arthritis \& Rheumatism. 2006;54.2:421-32.

10. Popescu A, Kao AH. Neuropsychiatric systemic lupus erythematosus. Curr Neuropharmacol. 2011;9(3):449-57. Epub 2012/03/02

11. Bertsias GK, Ioannidis JP, Aringer M, Bollen E, Bombardieri S, Bruce IN, et al. EULAR recommendations for the management of systemic lupus erythematosus with neuropsychiatric manifestations: report of a task force of the EULAR standing committee for clinical affairs. Ann Rheum Dis. 2010;69(12):207482. Epub 2010/08/21

12. Miyakis S, Lockshin MD, Atsumi T, Branch DW, Brey RL, Cervera R, et al. International consensus statement on an update of the classification criteria for definite antiphospholipid syndrome (APS). Journal of thrombosis and haemostasis. 2006;4(2):295-306. Epub 2006/01/20

13. Tektonidou MG, et al. EULAR recommendations for the management of antiphospholipid syndrome in adults. Annals of the rheumatic diseases. 2019; annrheumdis-2019

14. Bloch DA, et al. The American College of Rheumatology 1990 criteria for the classification of vasculitis: patients and methods. Arthritis \& Rheumatism. 1990;33.8:1068-73.

15. Jennette JC, et al. 2012 revised international chapel hill consensus conference nomenclature of vasculitides. Arthritis \& Rheumatism. 2013;65.1:1-11.

16. Abdulkader R, et al. Classification of vasculitis: EMA classification using CHCC 2012 definitions. Annals of the rheumatic diseases. 2013;72.11:1888.

17. Yates M, et al. EULAR/ERA-EDTA recommendations for the management of ANCA-associated vasculitis. Annals of the rheumatic diseases. 2016;75:1583-94.

18. Dasgupta B, Cimmino MA, Kremers HM, Schmidt WA, Schirmer M, Salvarani C, et al. 2012 Provisional classification criteria for polymyalgia rheumatica: a European League Against Rheumatism/American 
College of Rheumatology collaborative initiative. Arthritis and rheumatism. 2012;64(4):943-54.

19. Dejaco C, et al. 2015 Recommendations for the management of polymyalgia rheumatica: a European League Against Rheumatism/American College of Rheumatology collaborative initiative. Arthritis \& rheumatology. 2015;67.10:2569-80.

20. Rudwaleit M, van der Heijde D, Landewe R, Akkoc $\mathrm{N}$, Brandt J, Chou CT, et al. The assessment of SpondyloArthritis international society classification criteria for peripheral spondyloarthritis and for spondyloarthritis in general. Ann Rheum Dis. 2011;70(1):25-31. Epub 2010/11/27

21. Sieper J, et al. New criteria for inflammatory Back pain in patients with chronic back pain: a real patient exercise by experts from the assessment of Spondyloarthritis international society (ASAS). Ann Rheum Dis. 2009;68:784-8.

22. van der Heijde, Désirée, et al. 2016 update of the ASAS-EULAR management recommendations for axial spondyloarthritis. Annals of the rheumatic diseases. 2017;76.6:978-91.

23. Congi L, Roussou E. Clinical application of the CASPAR criteria for psoriatic arthritis compared to other existing criteria. Clin Exp Rheumatol. 2010;28(3):304-10.

24. Gossec L, et al. European League Against Rheumatism (EULAR) recommendations for the management of psoriatic arthritis with pharmacological therapies: 2015 update. Annals of the rheumatic diseases. 2016;75.3:499-510.

25. van den Hoogen F, Khanna D, Fransen J, Johnson SR, Baron M, Tyndall A, et al. 2013 classification criteria for systemic sclerosis: an American college of rheumatology/European league against rheumatism collaborative initiative. Ann Rheum Dis. 2013;72(11):1747-55. Epub 2013/10/05

26. Kowal-Bielecka O, et al. Ann Rheum Dis. 2017;76:1327-39. https://doi.org/10.1136/ annrheumdis-2016-209909.

27. Raychaudhuri SP, Mitra A. Polymyositis and dermatomyositis: disease spectrum and classification. Indian J Dermatol. 2012;57(5):366.

28. Shiboski CH, et al. 2016 ACR-EULAR Classification Criteria for primary Sjögren's Syndrome: A Consensus and Data-Driven Methodology Involving Three International Patient Cohorts. Arthritis \& rheumatology (Hoboken, NJ). 2017;69.1:35.

29. Vivino FB, et al. New treatment guidelines for Sjögren's disease. Rheum Dis Clin N Am. 2016;42(3):531-51. https://doi.org/10.1016/j.rdc.2016.03.010.

30. Hatemi $G$, et al. EULAR recommendations for the management of Behçet disease. Annals of the rheumatic diseases. 2008;67.12:1656-62.
31. Sivera F, Andrés M, Carmona L, et al. Multinational evidence-based recommendations for the diagnosis and management of gout: integrating systematic literature review and expert opinion of a broad panel of rheumatologists in the $3 \mathrm{e}$ initiative. Ann Rheum Dis. 2014;73:328-35.

32. Altman R, et al. Development of criteria for the classification and reporting of osteoarthritis: classification of osteoarthritis of the knee. Arthritis \& Rheumatism. 1986;29.8:1039-49.

33. Altman R, et al. The American College of Rheumatology criteria for the classification and reporting of osteoarthritis of the hand. Arthritis \& Rheumatism. 1990;33.11:1601-10.

34. Altman R, et al. The American College of Rheumatology criteria for the classification and reporting of osteoarthritis of the hip. Arthritis \& Rheumatism. 1991;34.5:505-14.

35. Hochberg MC, et al. American College of Rheumatology 2012 recommendations for the use of nonpharmacologic and pharmacologic therapies in osteoarthritis of the hand, hip, and knee. Arthritis care \& research. 2012;64.4:465-74.

36. Genant HK, Cooper C, Poor G, Reid I, Ehrlich G, Kanis J, Nordin BC, Barrett-Connor E, Black D, Bonjour JP, Dawson-Hughes B. Interim report and recommendations of the World Health Organization task-force for osteoporosis. Osteoporos Int. 1999;10(4):259-64.

37. Kanis JA, Borgstrom F, De Laet C, Johansson H. Assessment of fracture risk. OsteoporosInt. 2005;16:581-9.

38. Shepstone L, Lenaghan E, Cooper C, Clarke S, Fong-soe-khioe R, Fordham R, Gittoes N, Harvey I, Harvey N, Heawood A and Holland R, 2018. Articles Screening in the community to reduce fractures in older women (SCOOP): a randomised controlled trial.

39. Cosman F, de Beur SJ, LeBoff MS, Lewiecki EM, Tanner B, Randall S, Lindsay R. Clinician's guide to prevention and treatment of osteoporosis. Osteoporos Int. 2014;25(10):2359-81.

40. Panel on Prevention of Falls in Older Persons, American Geriatrics Society and British Geriatrics Society. Summary of the updated American Geriatrics Society/British geriatrics society clinical practice guideline for prevention of falls in older persons. J Am Geriatr Soc. 2011;59(1):148-57.

41. Briot K, Roux C, Thomas T, Blain H, Buchon D, Chapurlat R, Debiais F, Feron JM, Gauvain JB, Guggenbuhl P, Legrand E. 2018 update of French recommendations on the management of postmenopausal osteoporosis. Joint Bone Spine. 2018;85(5):519-30. 
Open Access This chapter is licensed under the terms of the Creative Commons Attribution 4.0 International License (http://creativecommons.org/licenses/by/4.0/), which permits use, sharing, adaptation, distribution and reproduction in any medium or format, as long as you give appropriate credit to the original author(s) and the source, provide a link to the Creative Commons license and indicate if changes were made.

The images or other third party material in this chapter are included in the chapter's Creative Commons license, unless indicated otherwise in a credit line to the material. If material is not included in the chapter's Creative Commons license and your intended use is not permitted by statutory regulation or exceeds the permitted use, you will need to obtain permission directly from the copyright holder.

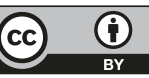

